\title{
Role of Aedes aegypti (Linnaeus) and Aedes albopictus (Skuse) in local dengue epidemics in Taiwan
}

\author{
Pui-Jen Tsai ${ }^{*}$ (1) and Hwa-Jen Teng ${ }^{2}$
}

\begin{abstract}
Background: Aedes mosquitoes in Taiwan mainly comprise Aedes albopictus and Ae. aegypti. However, the species contributing to autochthonous dengue spread and the extent at which it occurs remain unclear. Thus, in this study, we spatially analyzed real data to determine spatial features related to local dengue incidence and mosquito density, particularly that of Ae. albopictus and Ae. aegypti.

Methods: We used bivariate Moran's I statistic and geographically weighted regression (GWR) spatial methods to analyze the globally spatial dependence and locally regressed relationship between (1) imported dengue incidences and Breteau indices (Bls) of Ae. albopictus, (2) imported dengue incidences and Bl of Ae. aegypti, (3) autochthonous dengue incidences and $\mathrm{BI}$ of Ae. albopictus, (4) autochthonous dengue incidences and $\mathrm{Bl}$ of Ae. aegypti, (5) all dengue incidences and Bl of Ae. albopictus, (6) all dengue incidences and BI of Ae. aegypti, (7) BI of Ae. albopictus and human population density, and (8) Bl of Ae. aegypti and human population density in 348 townships in Taiwan.
\end{abstract}

Results: In the GWR models, regression coefficients of spatially regressed relationships between the incidence of autochthonous dengue and vector density of Ae. aegypti were significant and positive in most townships in Taiwan. However, Ae. albopictus had significant but negative regression coefficients in clusters of dengue epidemics. In the global bivariate Moran's index, spatial dependence between the incidence of autochthonous dengue and vector density of Ae. aegypti was significant and exhibited positive correlation in Taiwan (bivariate Moran's index $=0.51$ ). However, Ae. albopictus exhibited positively significant but low correlation (bivariate Moran's index $=0.06$ ). Similar results were observed in the two spatial methods between all dengue incidences and Aedes mosquitoes (Ae. aegypti and Ae. albopictus).

The regression coefficients of spatially regressed relationships between imported dengue cases and Aedes mosquitoes (Ae. aegypti and Ae. albopictus) were significant in 348 townships in Taiwan. The results indicated that local Aedes mosquitoes do not contribute to the dengue incidence of imported cases.

The density of Ae. aegypti positively correlated with the density of human population. By contrast, the density of Ae. albopictus negatively correlated with the density of human population in the areas of southern Taiwan. The results indicated that Ae. aegypti has more opportunities for human-mosquito contact in dengue endemic areas in southern Taiwan.

Conclusions: Ae. aegypti, but not Ae. albopictus, and human population density in southern Taiwan are closely associated with an increased risk of autochthonous dengue incidence.

Keywords: Dengue fever, Aedes mosquitoes, Breteau index, Global bivariate Moran's I, Geographically weighted regression

\footnotetext{
* Correspondence: puijentsai@gmail.com

${ }^{1}$ Center for General Education, Aletheia University, New Taipei City 25103,

Taiwan, ROC

Full list of author information is available at the end of the article
} 


\section{Background}

Dengue fever (DF) is a serious mosquito-borne viral infectious disease, mostly distributed in tropical regions [1]. DF epidemics were first recognized nearly simultaneously in Asia, Africa, and North America in the 1780s [2]. Later, DF epidemics were mostly reported in Southeast Asia, particularly after the end of World War II [3]. DF epidemics became more frequent and extended to Latin America in the early 1980s [4]. Dengue virus (DENV), with a nearly ubiquitous distribution in tropical regions, was more recently introduced in Europe [5]. In DENV circulation areas, 3.97 billion people are estimated to have a risk of dengue infections [6]. Moreover, approximately 50 million clinical cases annually occur in more than 100 countries worldwide [7]. Of these, $2.5 \%$ of dengue cases become more severe and progress into dengue hemorrhagic fever (DHF) and/or dengue shock syndrome (DSS). These severe forms of the disease are responsible for high morbidity and fatal outcomes. Approximately $5 \%$ of patients with DHF or DSS are estimated to die, predominantly children younger than 15 years old [8]. However, in recent years, severe dengue cases have tended to increase among adults in some areas [9]. DENV is the etiological agent of DF, DHF, and DSS. A sylvatic cycle that serves as an enzootic cycle involving monkeys and jungle mosquitoes may be present [10]. A study reported that the currently circulating DENV-1 may have resulted from a spillover of ancestral sylvatic strains [11]. DENV is one of the 70 members of the genus Flavivirus of the family Flaviviridae, which consists of four closely related but genetically distinct antigenic serotypes, namely DENV-1, -2, -3 , and -4, originally classified based on their serological characteristics [12]. Each DENV serotype elicits neutralizing antibodies in infected humans, resulting in lifelong immunity against the corresponding virus serotype [3]. DENV can infect various tissues in mosquito vectors, particularly the midgut and salivary glands [13].

The mosquitoes Aedes aegypti and Ae. albopictus [14] are vectors of several globally critical arboviruses including DENV [15], yellow fever virus [16], chikungunya virus [17], and Zika viruses [18]. Ae. aegypti originated in Africa, where its ancestral form was a zoophilic tree-hole mosquito named Ae. aegypti formosus [19]. The domestic form of Ae. aegypti is genetically distinct with discrete geographic niches [20]. It has been hypothesized that harsh conditions coupled with the onset of the slave trade resulted in the introduction of Ae. aegypti into the new world from Africa, from where it subsequently spread into tropical and subtropical regions of the world [19]. Ae. albopictus, originally a zoophilic forest species from Asia [21], spread to islands in the Indian and Pacific Oceans [22]. During the 1980s, Ae. albopictus rapidly expanded into Europe, the United States, Brazil, and Central Africa [23-25].

Taiwan is geographically located in a region that spans both tropical and subtropical climates $\left(22-25^{\circ} \mathrm{N}\right.$ and 120-122 $2^{\circ}$ ). Although both Ae. aegypti and Ae. albopictus are prevalent in Taiwan, they have different distributions [26, 27]. Ae. albopictus is extensively distributed at elevations of less than $1500 \mathrm{~m}$ throughout the island, whereas Ae. aegypti appears only in the south below the Tropic of Cancer (i.e., $23.5^{\circ} \mathrm{N}$ ). The Taiwan Centers for Disease Control (CDC) reported similar results based on surveys of the dengue vector Ae. aegypti conducted in Taiwan during 1988-1996 and 2003-2004 [27]. However, the reason behind the absence of Ae. aegypti in northern Taiwan remains unclear. This absence may be attributable to the sensitivity of Ae. aegypti to lower winter temperatures in the north, resulting in their limited distribution in Taiwan [28]. The same observations were reported in the temperate and subtropical regions of Argentina (e.g., the city of Resistencia, Chaco). The mortality rate of Ae. aegypti eggs due to lower winter temperatures was $48.6 \%$ [29]. DF is a travel-related disease in Taiwan because travelers can carry DENV from endemic areas into the island [30-33]. After the transport of this virus into the island, it is passed on to Aedes mosquitoes, which can cause an outbreak of DF. Historical epidemics of dengue in Taiwan were documented in 1902, 1915, and 1922 in Penghu Islet (i.e., Penghu County); 1924 and 1927 in southern Taiwan; 1931 in Tainan; and 1942-1943, island-wide [34]. A reason for these epidemics was the high prevalence of water storage tanks among households during wartime and the entry of dengue patients from epidemic or endemic countries into Southeast Asia. The virus was silent for almost 37 years until 1981, when the DEN-2 DF epidemic occurred on the islet of Hsiao-LiuChiu (i.e., Liouciou Township), which is located off southern Taiwan [35]. However, it did not cause an epidemic in mainland Taiwan. A DENV-1 epidemic occurred in 1987-1988 in southern Taiwan. In 1987-2002, the epidemic patterns of dengue in Taiwan cycled with small-scale outbreaks occurring nearly every 3 years and large-scale epidemics occurring nearly every 10 years [36]. However, since 2002, the Taiwan CDC have recorded nine outbreaks with more than 1000 cases of DF and/or DHF/DSS in 2002, 2006, 2007, 2009, 2010, 2011, 2012, 2014, and 2015, respectively [37]. With a few exceptions, these outbreaks occurred in the south of Taiwan where $A e$. aegypti is prevalent and coexists with Ae. albopictus. The relationship between vectors and autochthonous dengue incidence in terms of geographical correlation has not yet been quantitatively assessed. Therefore, the role of Aedes mosquitoes (i.e., Ae. aegypti and Ae. albopictus) in local dengue epidemics has largely been 
neglected in spatial research. Therefore, this study investigated the role of Aedes mosquitoes in the occurrence of autochthonous dengue and the effects of human population density on the distribution of Aedes mosquitoes in Taiwan.

\section{Methods}

\section{Surveillance of Aedes mosquitoes in the main island of} Taiwan

The mosquito data set used in this study was a part of national routine entomological surveillance of dengue and the original data collected in a 3-year project of Taiwan CDC through 2009-2011 [27]. In brief, a sample of 100 mosquito larvae was collected from each of the 7,019 subtownships (i.e., villages or communities; the smallest administrative units in this study) in the main island of Taiwan between 2009 and 2011. In the villages, the mosquito larvae sampling frequency ranged from 1 to 130 over the 3 years, and the mean sampling frequency was 23. All samples were sent to the CDC for laboratory analysis. All mosquito classification data from the 7,019 villages were categorized into 365 townships. In this study, we focused on the main island of Taiwan for detecting spatial contiguity and pattern consistency. Thus, we excluded townships belonging to outlying islands and investigated a total of 348 townships (Wutai Township, which is located in a remote mountainous area, was the only township on the main island of Taiwan that was not investigated; Fig. 1). The incidence rates of Ae. albopictus in each township were calculated using the number of confirmed Ae. albopictus larvae as a numerator and a total of confirmed Aedes larvae as a denominator. The incidence rates of Ae. aegypti were calculated using the same formula.

The Breteau index (BI) is defined as the number of positive containers per 100 houses inspected [38]. We used averaged BI values to represent the dengue vector density in a given area. BI data for the period between 2009 and 2011 were obtained from the CDC. A total of $115,690 \mathrm{BI}$ records for the villages were archived into 348 townships and were used for calculating the average BI. For each township, the BI of Ae. albopictus was calculated by multiplying the incidence rate of Ae. albopictus with the average $\mathrm{BI}$, and the $\mathrm{BI}$ of Ae. aegypti was calculated using the same formula.

\section{Data collection for confirmed dengue cases}

The data of confirmed imported and autochthonous DF cases were obtained from the Taiwan National Infectious Disease Statistics System of the CDC [37]. Because DF is a notifiable disease, blood samples from patients with suspected DF symptoms are collected and sent to the $\mathrm{CDC}$ for laboratory confirmation. Patients who had any of the following condition will be considered to have

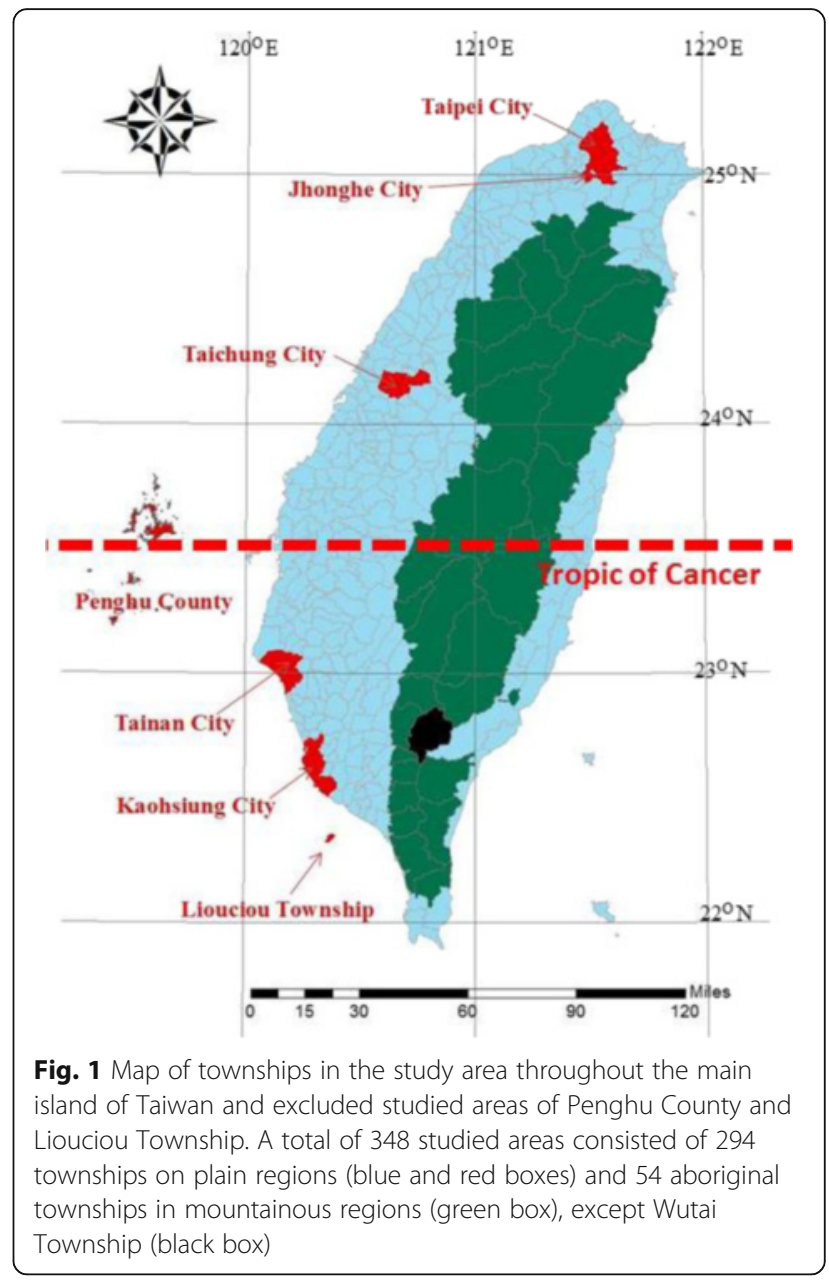

DENV infection: (i) positive virus isolation; (ii) positive result of real-time polymerase chain reaction; (iii) positive result of higher titers of dengue-specific IgM and IgG antibody in which cross-reaction to Japanese encephalitis had been excluded; or (iv) positive seroconversion or 4-fold rise in dengue-specific IgM or IgG antibody in the convalescent phase and positive result in the NS1 antigen test [32, 39].

Data were obtained for the period between 2009 and 2011. According to the definition of the CDC, epidemiological questions regarding the travel history, incubation period, and first day of illness of patients confirmed as having DF were used to identify the possible origin of dengue infection. Laboratory-confirmed dengue cases with a travel history to endemic countries within 14 days before the date of dengue onset were defined as "dengue cases imported to Taiwan" [39]. The remaining confirmed dengue cases were defined as autochthonous cases. Ethical approval for this study was not required because we used public domain data. We evaluated 348 townships on the main island of Taiwan. The demographic data used in this study were obtained from the 
Ministry of the Interior [40]. Between 2009 and 2011, the incidence rate of imported and autochthonous dengue cases and the average ratio of all confirmed dengue cases were calculated for each township [37]. The incidence rate was calculated as the number of confirmed dengue cases divided by the total human population in that township.

\section{Global Moran's I Statistic}

Global Moran's spatial autocorrelation was used to assess the correlation among neighboring observations and to identify patterns and levels of spatial clustering in neighboring districts [41]. Moran's statistic [42], similar to the Pearson correlation coefficient, was calculated using the following formula:

$$
I=\frac{N}{S_{O}} \sum_{i} \sum_{j} w_{i j} \frac{\left(x_{i}-\bar{x}\right)\left(x_{j}-\bar{x}\right)}{\sum_{i}\left(x_{i}-\bar{x}\right)^{2}}
$$

where $N$ is the number of districts, $w_{i j}$ is the element in the spatial weight matrix corresponding to the observation pair $i$ and $j$, and $x_{i}$ and $x_{j}$ observations for the areas $i$ and $j$, respectively, with the mean $\bar{x}$, and

$$
S_{O}=\sum_{i} \sum_{j} w_{i j}
$$

Because weights were row standardized $\left(\sum w_{i j}=1\right)$, the first step in the spatial autocorrelation analysis was to construct a spatial weight matrix that contained information on the neighborhood structure for each location. Adjacency was defined as immediately neighboring administrative districts, including the district itself. Non-neighboring administrative districts were assigned a weight of zero. Spatial contiguity for polygons was defined as the property of sharing a common boundary or vertex. Contiguity analysis is a crucial method for assessing unusual features in connectivity distribution [43]. Queen's contiguity can be used to compensate for spatial contiguity by incorporating both the Rook and Bishop relationships into a single measure [44]. The administrative districts considered in this study were highly irregular in both shape and size. The first-order queen polygon contiguity method was the most appropriate for quantifying the spatial weights matrix for the analysis of connectivity. On the basis of this approach, spatial weight or connectivity matrices were determined and used in conjunction with the global Moran's calculations described further.

Moran's I values may range from -1 (dispersed) to +1 (clustered). A Moran's I value of 0 suggests complete spatial randomness. A random permutation procedure recalculates a statistic many times by reshuffling data values among map units to generate a reference distribution. The obtained calculated statistic, based on the observed spatial pattern, is then compared to the reference distribution, and a pseudo significance level (pseudo $p$ value) is computed. To verify that the Moran's I value significantly differed from the expected value, we used a Monte Carlo randomization test with 9,999 permutations to obtain significant values. The data values were reassigned among the $\mathrm{N}$ locations, providing a randomized distribution against which the observed value could be judged. If the observed I value was within the tails of this distribution, a significant spatial autocorrelation was present in the data and a pseudo $p$ value was smaller than 0.05; thus, the assumption of independence among the observations could be rejected [45].

\section{Global bivariate Moran's I statistic}

The global bivariate Moran's I statistic quantifies the spatial dependency between the two variables $X_{1}$ and $X_{k}$ in a same location $i$ [46]. This yields a counterpart of a univariate Moran-like spatial autocorrelation, defined as follows:

$$
I_{k l}=\frac{Z_{k} W Z_{l}}{n}
$$

Where $n$ is the number of observations, $z_{k}=\left[x_{k}-\bar{x}_{k}\right] / \sigma_{k}$ and $z_{l}=\left[x_{l}-\bar{x}_{l}\right] / \sigma_{l}$, which have been standardized such that the mean is zero and standard deviation equals one. $W$ is the row-standardized spatial weight matrix. The weight matrix defines the neighbor set for each observation with non-zero elements for neighbors and zero for the others. The significance of this bivariate spatial correlation can be typically assessed using a randomization (or permutation) approach.

In this case, observed values for one of the variables are randomly reallocated to locations (centroids of townships), and the statistic is recomputed for each such random pattern. The resulting empirical reference distribution can aid in evaluating how extreme the observed statistic is relative to its distribution under spatial randomness to produce a Moran's I scatter plot. The Moran's I scatter plot visualizes a spatial autocorrelation statistic as the slope of the regression line with the spatial lag (a weighted average of the value of a variable in the neighboring locations) on the vertical axis and the original variable on the horizontal axis [46].

The same analysis is true for the bivariate Moran's I scatter plot. The slope of the linear regression through this scatter plot equals the statistic in equ. (3), yielding an interpretation of the spatial lag because inverse distance neighboring values will be used for the spatial weight matrix.

Because the $\mathrm{Z}$ variables are standardized, the sum of squares used in the denominator of equ. (3) is constant and is equivalent to $n$, irrespective of whether $Z_{k}$ or $Z_{l}$ is used. Therefore, the focus is on the linear association of 
a variable $Z_{k}$ at a location $\mathrm{i}\left(\mathrm{Z}_{\mathrm{ki}}\right)$ with the corresponding spatial lag of the other variable $\left[\mathrm{W}_{\mathrm{zl}}\right]_{\mathrm{i}}$. This concept was derived from bivariate spatial correlation and therefore centers on the extent to which values of the variable $Z_{k}$ observed at a given location $\mathrm{k}$ exhibit a systematic association with another variable $\mathrm{Z}_{\mathrm{l}}$ observed at the neighboring location $i$ [47].

Global bivariate Moran's I values may range from -1 to +1 . A Moran's I value of $-1,0$, and 1 indicates perfect negative spatial dependence between variables, no correlation between variables, and perfect positive spatial dependence between variables, respectively. A random permutation procedure recalculates a statistic numerous times by reshuffling data values among map units to generate a reference distribution. The obtained calculated statistic based on the observed spatial pattern was then compared to this reference distribution, and a pseudo significance level (pseudo $p$ value) was computed. To verify that the Moran's I value significantly differed from the expected value, we applied a Monte Carlo randomization test with 9,999 permutations to obtain significant values. Data values were reassigned among the $\mathrm{N}$ locations, providing a randomized distribution against which the observed value may be judged. If the observed Moran's I value was within the tails of this distribution, significant spatial dependence was present in the data, and if there the pseudo $p$ value was less than 0.05 , then the assumption of independence among the observations could be rejected. Global Moran's I statistic and Global bivariate Moran's I statistic were calculated using GeoDa 1.4.6 [48].

\section{Geographically weighted regression}

Geographically weighted regression (GWR) is a type of a local statistic that can produce a set of local parameter estimates demonstrating the variation of a relationship over space and then enable examination of the spatial pattern of the local estimates to gain some understanding of possible hidden causes for the pattern. GWR provides a local model of the variable or process by fitting a regression equation to every feature in the dataset. GWR constructs these separate equations by incorporating the response and explanatory variables of features falling within the bandwidth of each target feature. The shape and size of the bandwidth is dependent on the user input for the kernel type, bandwidth method, distance, and number of neighbor parameters [49].

GWR [49, 50] extends the traditional regression framework (such as the ordinary least squares model; OLS) by enabling local, rather than global, parameters to be estimated so that the model is rewritten as follows:

$$
y_{i}=\beta_{o}\left(u_{i}, v_{i}\right)+\sum_{j} \beta_{j}\left(u_{i}, v_{i}\right) x_{i j}+\varepsilon_{i}
$$

where $y_{i}$ is the response variable, $u_{i}$ and $v_{i}$ are the coordinates for each location $i, \beta_{0}\left(\mathrm{u}_{\mathrm{i}}, \mathrm{v}_{\mathrm{i}}\right)$ is the intercept for location $i, \beta_{\mathrm{j}}\left(\mathrm{u}_{\mathrm{i}}, \mathrm{v}_{\mathrm{i}}\right)$ is the continuous function $\beta_{\mathrm{j}}(u, v)$ at location $i, \mathrm{x}_{\mathrm{ij}}$ is the ith observation of attribute $\mathrm{x}$ at location $j$, and $\varepsilon_{\mathrm{i}}$ is the error term for location $i$.

The weight assigned to each observation is based on a distance-decay function centered on observation $i$.

The estimator for the GWR model is similar to the weighted least squares global model, except that the weights are conditioned on location $u$ relative to the observations in the dataset and thus change for each location. The estimator is expressed as follows:

$$
\hat{\beta}(u)=\left(X^{T} W(u) X\right)^{-1} X^{T} W(u) y
$$

$W(u)$ is a square matrix of weights relative to the position $u$. A particular location can be indexed $\left(\mathrm{u}_{\mathrm{j}}, \mathrm{v}_{\mathrm{j}}\right)$ in the study area. $X T W(u) X$ is the geographically weighted variance-covariance matrix, and $y$ is the vector of the response variable value.

The $W(u)$ matrix contains the geographical weights in its leading diagonal and zero in its off-diagonal elements.

$$
\left[\begin{array}{cccc}
w_{1}(u) & 0 & 0 & 0 \\
0 & w_{2}(u) & 0 & 0 \\
0 & 0 & \cdots & 0 \\
0 & 0 & 0 & w_{n}(u)
\end{array}\right]
$$

The distance-decay function, which may take various forms, is modified by a bandwidth setting at a distance at which the weight rapidly approaches zero. In the area in which the present study was conducted, the sample points raised from the polygon centroids were not regularly placed, but were clustered. A convenient way of implementing the adaptive bandwidth specification is to select a kernel that allows the same number of sample points for estimations.

The weight can be calculated using the fixed spatial kernel and the value set for any observation with a distance exceeding the bandwidth to zero. The bi-square function can be expressed as follows:

$$
w_{i j}=\left(1-\left(d_{i j} / h\right)^{2}\right)^{2}
$$

where $\mathrm{w}_{\mathrm{ij}}$ is a continuous function of $\mathrm{d}_{\mathrm{ij}}, \mathrm{d}_{\mathrm{ij}}$ is the distance from the location $i$ to $j, \mathrm{w}_{\mathrm{ij}}$ is zero when $\mathrm{d}_{\mathrm{ij}}>h$ ( $h$ represents a quantity known as the bandwidth). This is a near-Gaussian function with the useful property of the weight being zero at a finite distance.

When the values for a particular explanatory variable cluster spatially, problems with local multicollinearity 
a

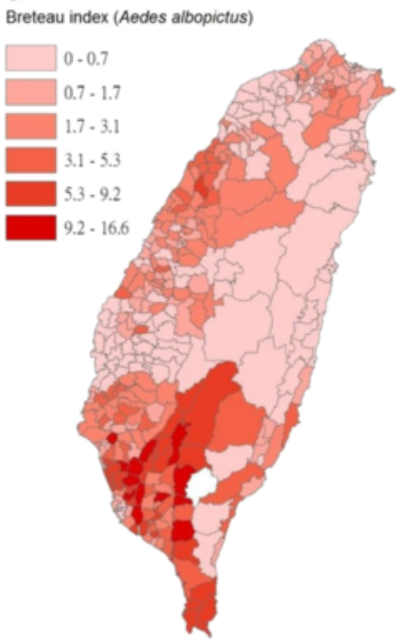

d

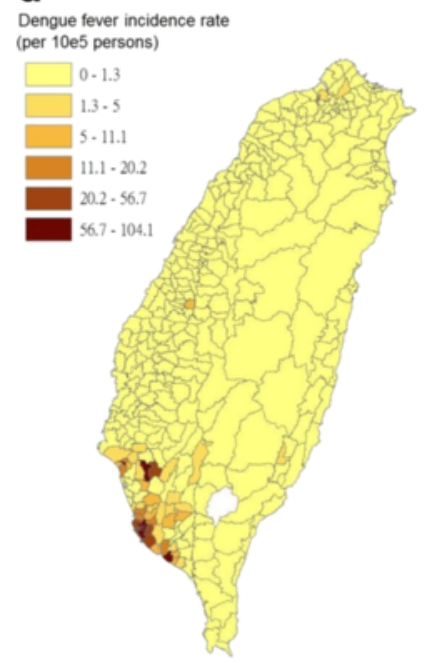

g

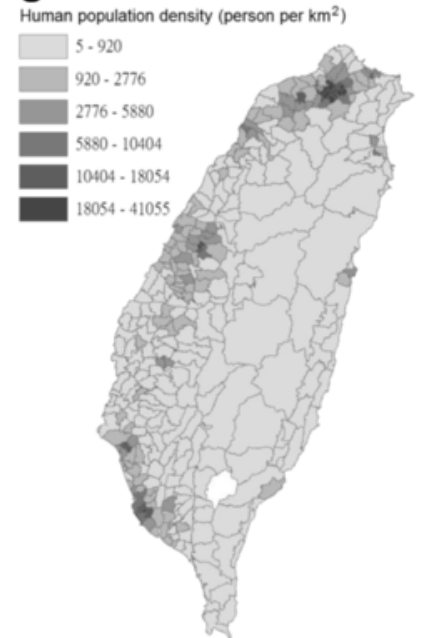

b

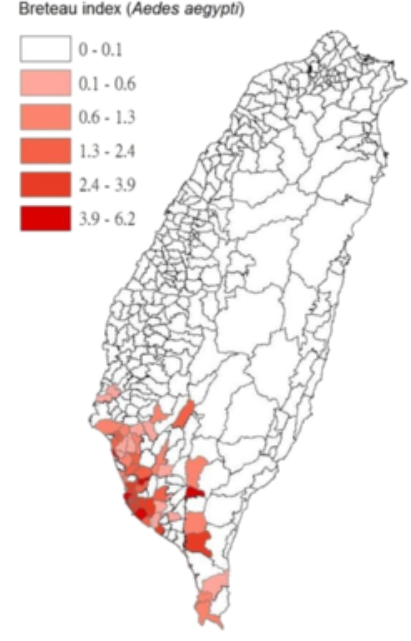

e

Autochthonous dengue incidence rate (per $10 \mathrm{e} 5$ persons)

$\square .1,3$

$\square+3 \cdot 5$

$11.1 \cdot 20.2$

$20.2 \cdot 56.7$

$56.7 \cdot 104.1$

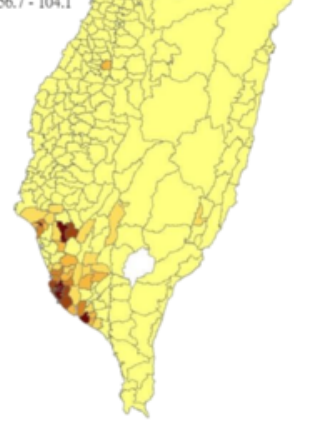

C

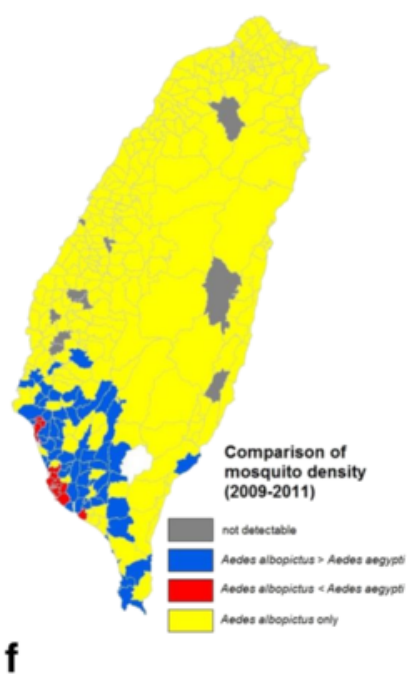
Imported dengue incidence rate (per $10 \mathrm{e} 5$ persons)

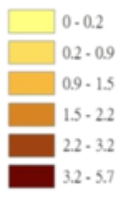

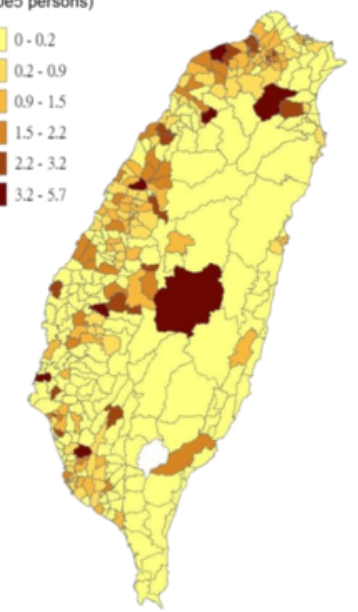

Fig. 2 (See legend on next page.) 
(See figure on previous page.)

Fig. 2 Maps of denque fever incidence, Aedes mosquito density, and human population density in 348 townships in Taiwan during 2009-2011. a Breteau index (BI) of Ae. albopictus during 2009-2011. b Bl of Ae. aegypti during 2009-2011. c Comparison between Ae. albopictus and Ae. aegypti density. d Incidence rates of all dengue fever (DF) cases during 2009-2011. e Incidence rates of autochthonous DF cases during 2009-2011. f Incidence rates of imported DF cases during 2009-2011. g Human population density during 2009-2011

likely occur. The condition number in the output feature class indicates when results are unstable due to local multicollinearity. In general, results of features having a condition number of $>30$ should be interpreted with caution. Problems with local collinearity can prevent both the Akaike information criterion (AIC) and cross validation $(\mathrm{CV})$ bandwidth methods from resolving an optimal distance or number of neighbors.

The bandwidth was chosen by minimizing the AIC score, calculated as follows:

$$
A I C_{c}=2 n \log _{e}(\hat{\sigma})+n \log _{e}(2 \pi)+n\left\{\frac{\mathrm{n}+\operatorname{tr}(\mathrm{S})}{\mathrm{n}-2-\mathrm{yr}(\mathrm{S})}\right\}
$$

where $n$ is the sample size, $\hat{\sigma}$ is the estimated standard deviation of the error, and $\operatorname{tr}(S)$ is the trace of the hat matrix. The AIC method has the advantage of accounting for variation in the degrees of freedom among models centered on different observations. The optimal bandwidth was determined by minimizing the corrected AIC, as described by Fotheringham et al. [49].

GWR models produce a set of local regression results, including local intercepts, regression coefficients, residuals, $R$ squared, and condition numbers, which can be mapped to show their spatial variability. GWR models were employed and mapped using ArcMap 10.

In a global model, whether regression coefficients significantly differ from zero is typically examined. This can be accomplished using a $t$ test: the $t$ statistics and their associated $p$ values. A coefficient whose estimated value is found to be not significantly different from zero is associated with a variable whose variation does not contribute to the mode. Variables with nonsignificant regression coefficients can be eliminated from the model. The situation with GWR is slightly more complex. If one set of coefficients is associated with each regression point and with one set of standard errors, then potentially hundreds or thousands of tests would be required to determine whether coefficients are locally significant. The assumption behind the tests indicates that five in every hundred tests would be significant if a significance level of 0.05 is used [51].

The Benjamini-Hochberg (B-H) procedure for controlling the false discovery rate (FDR), which consistently modifies the significance level for each test, was suitably used to test the significance of local regression coefficients of GWR [51, 52]. Thissen et al. (2002) reported a rapid and easy method to calculate the FDR of the B-H procedure by using Microsoft Excel [52]. The B-H approach controls the FDR by sequentially comparing the observed $p$ value for each family of multiple test statistics, in order from the largest to the smallest, to a list of computed $\mathrm{B}-\mathrm{H}$ critical values $[\mathrm{pB}-\mathrm{H}(\mathrm{i})]$. The critical value on the list is determined for each test statistic, indexed by $i$, by linear interpolation between $\alpha / 2$ (for the largest observed $p$ value) to $(\alpha / 2) / m$, where $m$ is the family size (for the smallest of the $p$ values). The last value is the Bonferroni critical value; thus, the reason for the gain in power of $\mathrm{B}-\mathrm{H}$ relative to Bonferroni is clear. In the $\mathrm{B}-\mathrm{H}$ approach, only the smallest of the $m$ observed $p$ values are compared with the Bonferroni critical value. All other $p$ values are calculated using less stringent criteria [52]. The local regression coefficient is estimated to be significant if the $p$ value is less than the $\mathrm{B}-\mathrm{H}$ critical value; otherwise, it is deemed nonsignificant. The results of the significant determination of local regression coefficients were mapped using ArcMap 10.

\section{General statistics}

We used Yate's correction for chi-square tests [53] to analyze the variation in the incidence pattern (imported and autochthonous cases) among the 9 dengue outbreak years.

\section{Results}

Figure 2 presents a map showing the geographical distribution of the BIs of Ae. albopictus and Ae. aegypti per district; comparison of the density of Ae. albopictus and Ae. aegypti; incidence rates of DF, autochthonous dengue, and imported dengue cases; and density of human population between 2009 and 2011.

Table 1 lists the results of the global autocorrelation statistics for the variables of BI of Ae. albopictus; BI of Ae. aegypti; incidence rates of $\mathrm{DF}$, autochthonous dengue, and imported dengue cases; and density of human population. The results of the global Moran's test for all the variables were statistically significant, having a pseudo $p$ value of less than 0.05 and indicating spatial heterogeneity (clustered).

According to the results of the global autocorrelation statistics, all the studied variables indicated spatial heterogeneity, which refers to the uneven distribution of a trait, event, or relationship across a region. The research methods of spatial dependence (e.g., global bivariate Moran's I analysis) and spatial regression (e.g., GWR) were examined to investigate the relationship between 
Table 1 Global autocorrelation analysis of the studied variables in Taiwan during 2009-2011

\begin{tabular}{lcccc}
\hline Variable & Moran's index & Z(l) & Pseudo $p$ value & Description \\
\hline Aedes albopictus (Breteau Index) & 0.5718 & 17.46 & 0.0001 & Clustered \\
Aedes aegypti (Breteau Index) & 0.5818 & 18.03 & 0.0001 & Clustered \\
dengue fever incidence rate & 0.5587 & 17.75 & 0.0001 & Clustered \\
autochthonous dengue incidence rate & 0.5587 & 17.65 & 0.0001 & 0.0002 \\
imported dengue incidence rate & 0.1475 & 4.499 & 0.0001 & Clustered \\
human population density & 0.6623 & 20.54 & Clustered
\end{tabular}

$\mathrm{Z}(\mathrm{I})$ a value greater than 1.96 is considered statistically significant

the variables of Aedes mosquitoes and dengue incidences as well as between human population density and Aedes mosquitoes, which were implemented using eight types of combinations (Table 2). Global bivariate Moran's I analysis was used to indicate variations in spatial distribution of data patterns and in cases where the correlation of two variables was investigated. The results of global bivariate Moran's I analysis are listed in Table 2, with significance values based on a permutation approach and a corresponding $p$ value of $<0.05$. Figure 3 depicts the corresponding global bivariate Moran's I scatter plot. The slopes of the regression line in eight scatter plots equaled to Moran's I indicator in Table 2 and differed from zero, indicating significant spatial correlation among the studied variables.

Condition numbers indicate when results are unstable because of local multicollinearity and the sensitivity of a linear equation solution to small changes in matrix coefficients. The condition number included in the GWR output indicates when local collinearity is a problem. A condition number of $>30$ is not reliable. In this study, condition numbers were calculated using the GWR analysis, as presented in Figs. 4f, 5f, $6 \mathrm{f}, 7 \mathrm{f}, 8 \mathrm{f}, 9 \mathrm{f}, 10 \mathrm{f}$ and $11 \mathrm{f}$. The values ranged from 1 to 7.5. All the outcomes of fitting the GWR models were reliable.

The maps in Fig. 4 are presented as local intercepts, local regression coefficients, significant B-H FDR values, local residuals, local $R^{2}$ values, and condition number values, in which the imported incidence of DF fits the GWR models with the explanatory variable of the BI of Ae. albopictus during 2009-2011. As presented in Fig. 4, regression coefficients of the incidence rate of Ae. albopictus were not significant in all study areas (local $R^{2}<$ 0.09). The results indicated that local Ae. albopictus did not contribute to imported dengue cases in the main island of Taiwan. We used bivariate Moran's I analysis to examine spatial dependence between the imported incidence of DF and $\mathrm{BI}$ of Ae. albopictus, and the results were negatively significant and demonstrated low correlation (bivariate Moran's index $=-0.07$; Table 2 and Fig. 3a).

The maps in Fig. 5 are presented as local intercepts, local regression coefficients, significant B-H FDR values, local residuals, local $R^{2}$ values, and condition number values, in which the imported incidence of DF fits the GWR models with the explanatory variable of the BI of Ae. aegypti during 2009-2011. As presented in Fig. 5, regression coefficients of the incidence rate of Ae. aegypti were not significant in all study areas (local $R^{2}<0.08$ ). The results indicated that local Ae. aegypti did not contribute to imported dengue cases in the main island of Taiwan. We used bivariate Moran's I analysis to examine spatial dependence between the imported incidence of DF and BI of Ae. aegypti, and the results exhibited no correlation (bivariate Moran's index = 0.03; Table 2 and Fig. 3b).

The maps in Fig. 6 are presented as local intercepts, local regression coefficients, significant B-H FDR values,

Table 2 Spatial dependence tests between the original variables and spatial lag as the second variables, which were calculated using global bivariate Moran's I analysis in Taiwan during 2009-2011

\begin{tabular}{|c|c|c|c|c|}
\hline Original variable & Spatial lag (second variable) & Bivariate Moran's I & Pseudo $p$-value & Spatial dependence \\
\hline Aedes albopictus (Breteau Index) & Imported dengue incidence rate & -0.07 & 0.0019 & Negative \\
\hline Aedes aegypti (Breteau Index) & Imported dengue incidence rate & 0.03 & 0.0985 & No correlation \\
\hline Aedes albopictus (Breteau Index) & Autochthonous dengue incidence rate & 0.06 & 0.0122 & Positive \\
\hline Aedes aegypti (Breteau Index) & Autochthonous dengue incidence rate & 0.51 & 0.0001 & Positive \\
\hline Aedes albopictus (Breteau Index) & Dengue fever incidence rate & 0.06 & 0.0139 & Positive \\
\hline Aedes aegypti (Breteau Index) & Dengue fever incidence rate & 0.51 & 0.0001 & Positive \\
\hline Human population density & Aedes albopictus (Breteau Index) & -0.12 & 0.0001 & Negative \\
\hline Human population density & Aedes aegypti (Breteau Index) & 0.21 & 0.0001 & Positive \\
\hline
\end{tabular}




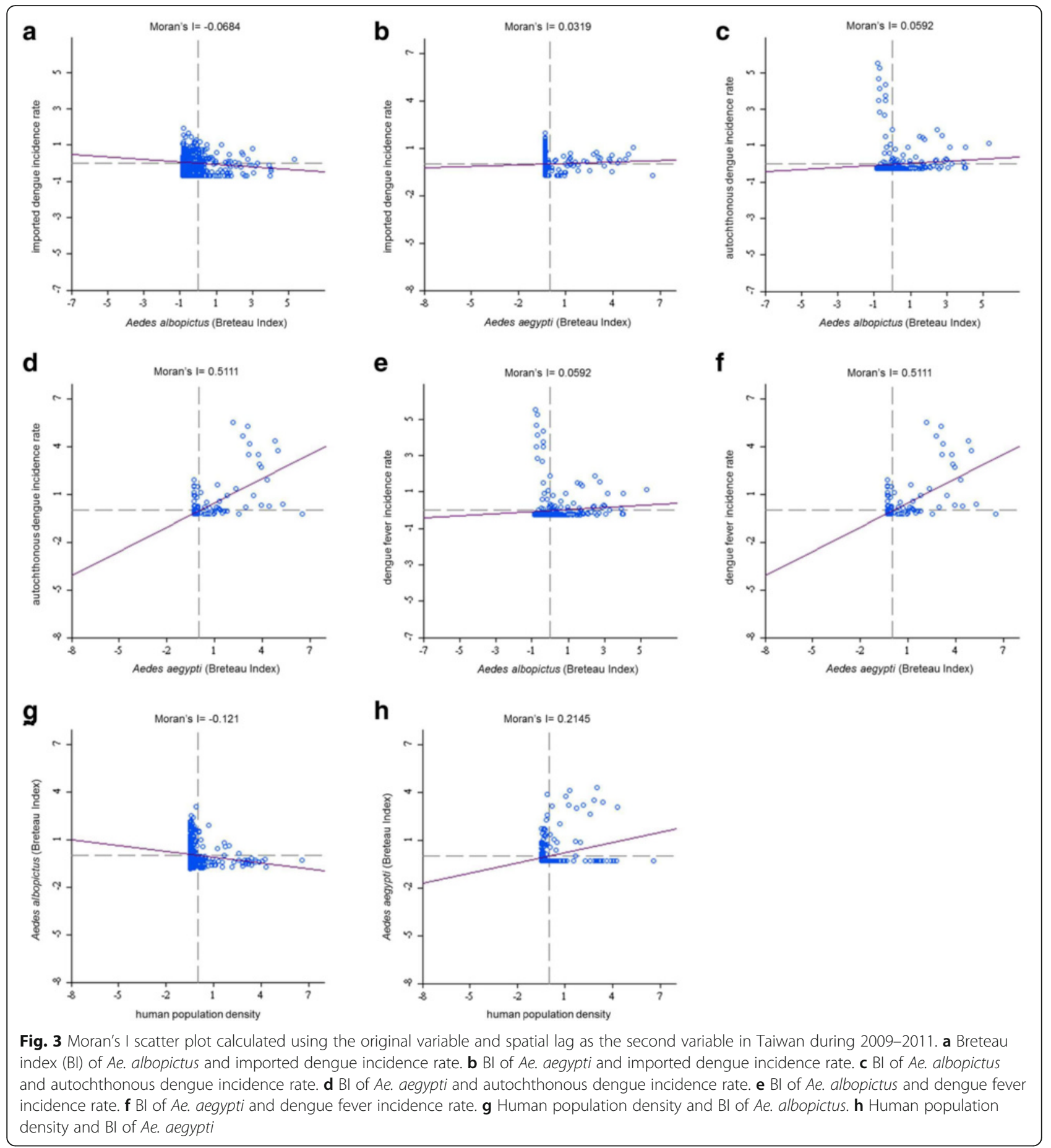

local residuals, local $R^{2}$ values, and condition number values, in which the autochthonous incidence of DF fits the GWR models with the explanatory variable of the BIs of Ae. albopictus during 2009-2011. As presented in Fig. 6, regression coefficients of the incidence rate of Ae. albopictus were negatively significant, with clusters covering most areas in the western area of southern Taiwan (local $R^{2}<0.45$ ). The results indicated that Ae. albopictus did not positively contribute to autochthonous dengue cases on the main island of Taiwan. We used bivariate Moran's I analysis to examine spatial dependence between the autochthonous incidence of DF and $\mathrm{BI}$ of Ae. albopictus, and the results were positively significant but exhibited low correlation (bivariate Moran's index = 0.06; Table 2 and Fig. 3c). 

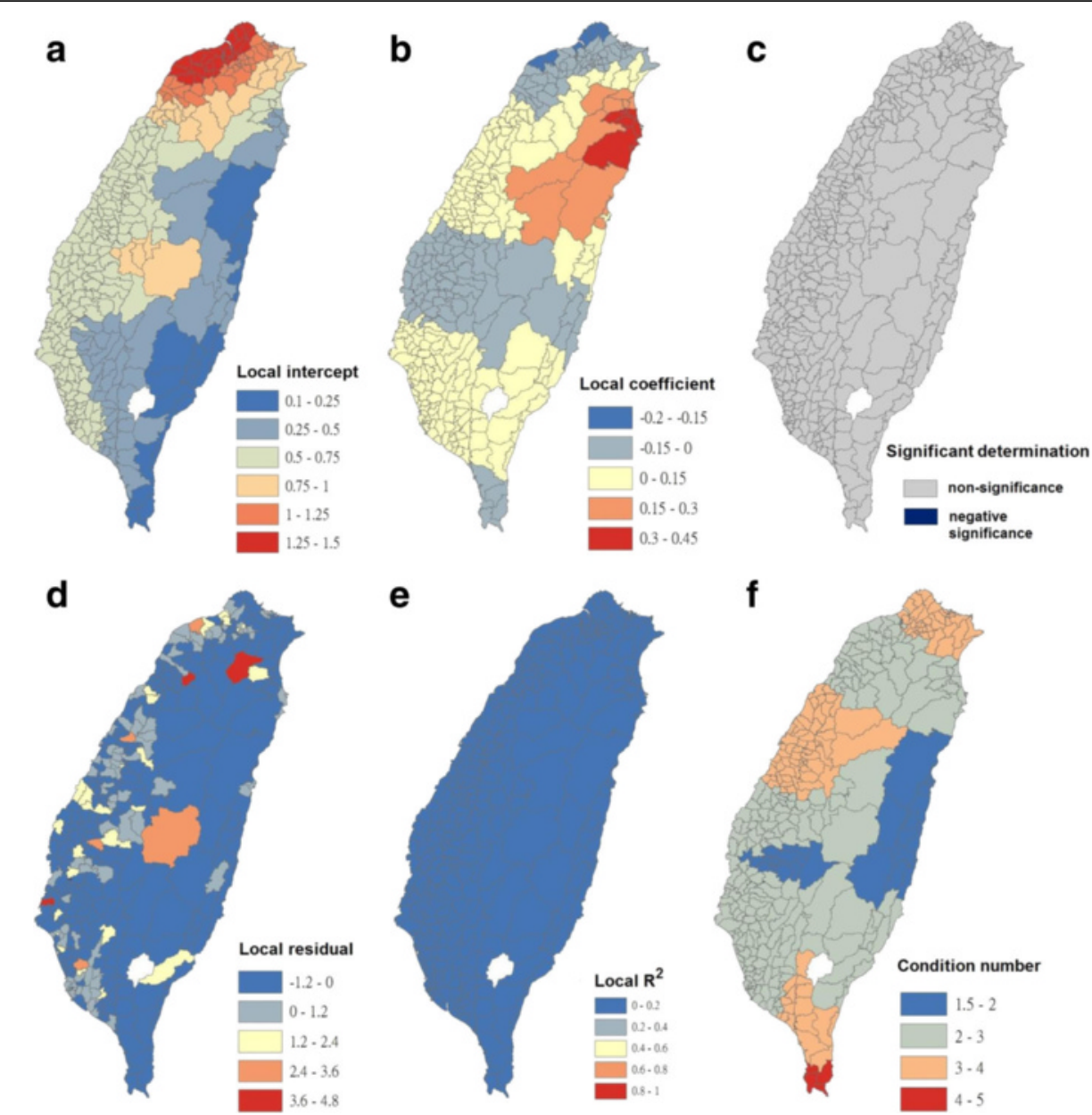

Fig. 4 Results of the GWR model for Breteau indices of Ae. albopictus (as the explanatory variable) and imported incidence of dengue fever (as the response variable) in 348 townships in Taiwan during 2009-2011. a Local intercepts during 2009-2011. b Local regression coefficients during 2009-2011. c Significant determination of coefficients according to the Benjamini-Hochberg false discovery rate during 2009-2011. d Local residuals during 2009-2011. e Local R² values during 2009-2011. f Local condition number values during 2009-2011

The maps in Fig. 7 are presented as local intercepts, local regression coefficients, significant B-H FDR values, local residuals, local $R^{2}$ values, and condition number values, in which the autochthonous incidence of DF fits the GWR models with the explanatory variable of the BIs of Ae. aegypti during 2009-2011. As presented in Fig. 7, regression coefficients of the incidence rate of Ae. aegypti were positively significant, with clusters covering most areas of Taiwan. A total of 290 townships correlated with local autochthonous dengue incidence (local $R^{2}<0.35$ ). The results indicated that autochthonous DF in Taiwan was transmitted by Ae. aegypti. Furthermore, the results of bivariate Moran's I analysis revealed that spatial dependence between the autochthonous incidence of DF and BI of Ae. aegypti was positively significant (bivariate Moran's index $=0.51$; Table 2 and Fig. 3d).

The maps in Fig. 8 are presented as local intercepts, local regression coefficients, significant B-H FDR values, local residuals, local $R^{2}$ values, and condition number values, in which the DF incidence fits the GWR models with the explanatory variable of the $\mathrm{BI}$ of Ae. albopictus during 2009-2011. As presented in Fig. 8, regression coefficients of the incidence rate of Ae. albopictus were negatively significant, with clusters covering most areas in the western area of southern Taiwan (local $R^{2}<0.45$ ). The results indicated that Ae. albopictus did not positively contribute to dengue cases in the main island of Taiwan. We used bivariate Moran's I analysis to examine spatial dependence between the incidence of DF and BI of Ae. albopictus, and the results were positively significant but had very low correlation (bivariate Moran's index $=0.06$; Table 2 and Fig. 3e).

The maps in Fig. 9 are presented as local intercepts, local regression coefficients, significant B-H FDR values, local residuals, local $R^{2}$ values, and condition number values, in which the incidence of DF fits the GWR models with the explanatory variable of the BI of 

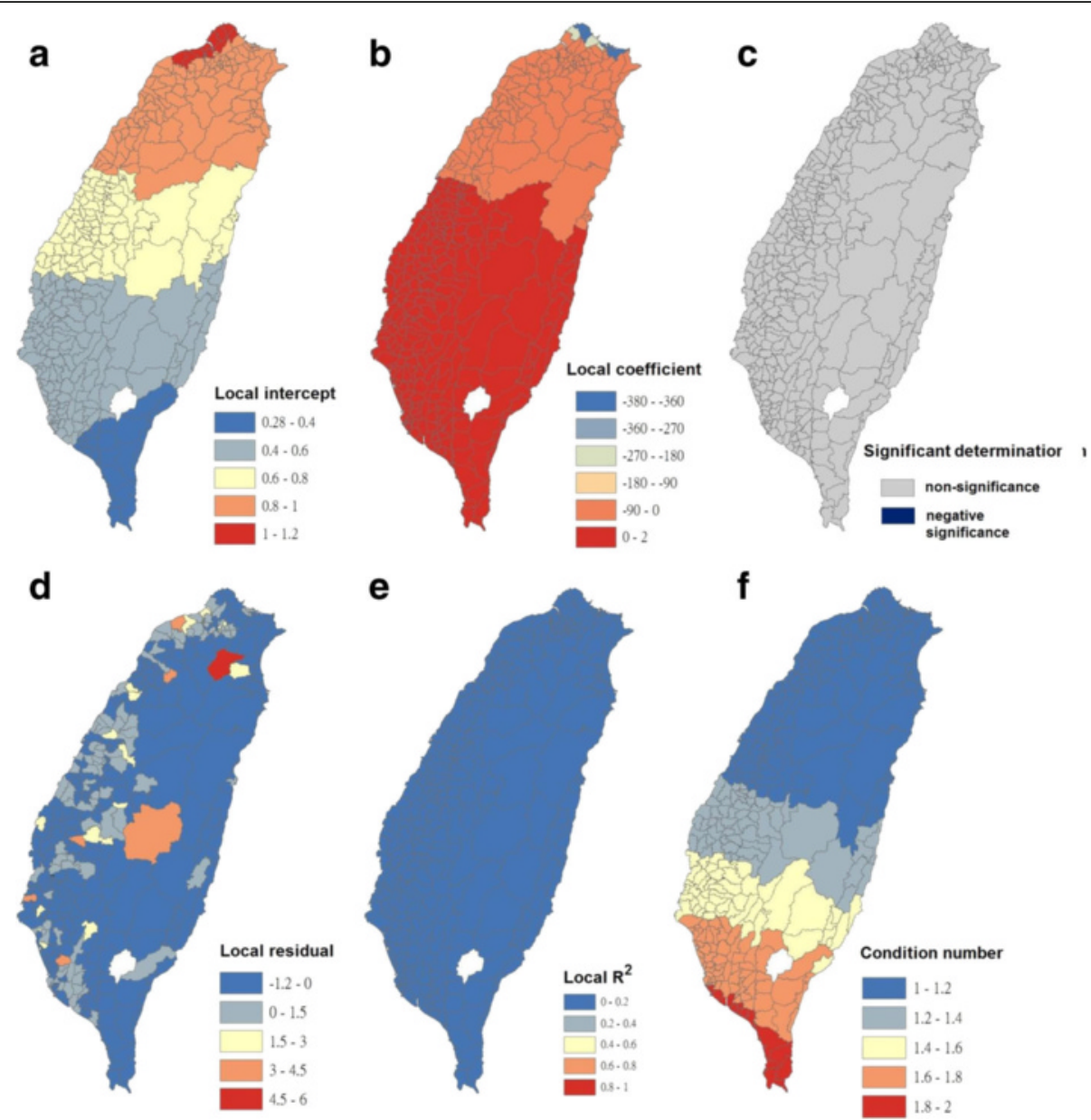

Fig. 5 Results of the GWR model for Breteau indices of Ae. aegypti (as the explanatory variable) and the imported incidence of dengue fever (as the response variable) in 348 townships in Taiwan during 2009-2011. a Local intercepts during 2009-2011. a Local regression coefficients during 2009-2011. c Significant determination of coefficients according to the Benjamini-Hochberg false discovery rate during 2009-2011. d Local residuals during 2009-2011. e Local R² values during 2009-2011. f Local condition number values during 2009-2011

Ae. aegypti during 2009-2011. As illustrated in Fig. 9, regression coefficients of the incidence rate of $A e$. aegypti were positively significant, with clusters covering most areas of Taiwan. A total of 290 townships were correlated with DF incidence (local $R^{2}<0.35$ ). The results indicated that DF incidence in Taiwan was transmitted by Ae. aegypti. We used bivariate Moran's I analysis to examine spatial dependence between the incidence of DF and $\mathrm{BI}$ of Ae. aegypti, and the results were positively significant (bivariate Moran's index = 0.51; Table 2 and Fig. 3f).

The maps in Fig. 10 are presented as local intercepts, local regression coefficients, significant B-H FDR values, local residuals, local $R^{2}$ values, and condition number values, in which the BI of Ae. albopictus fit the GWR models with the explanatory variable of human population density during 2009-2011. As presented in Fig. 10, regression coefficients of the human population density were negatively significant, with clusters covering most areas of plain and mountainous townships in the south part of Taiwan (local $R^{2}<0.5$ ). The results indicated that a high density of human population did not positively contribute to the prevalence of Ae. albopictus; however, it reduced the mosquito density in the south part of Taiwan. We used bivariate Moran's I analysis to examine spatial dependence between the BI of Ae. albopictus and density of human population, and the results were negatively significant (bivariate Moran's index $=-0.12$; Table 2 and Fig. 3g).

The maps in Fig. 11 are presented as local intercepts, local regression coefficients, significant B-H FDR values, local residuals, local $R^{2}$ values, and condition number values, in which the BI of Ae. aegypti fits the GWR models with the explanatory variable of the human population density during 2009-2011. As illustrated in Fig. 11, regression coefficients of the human population density were positively significant, with clusters covering most areas of Taiwan's townships in the south of the 

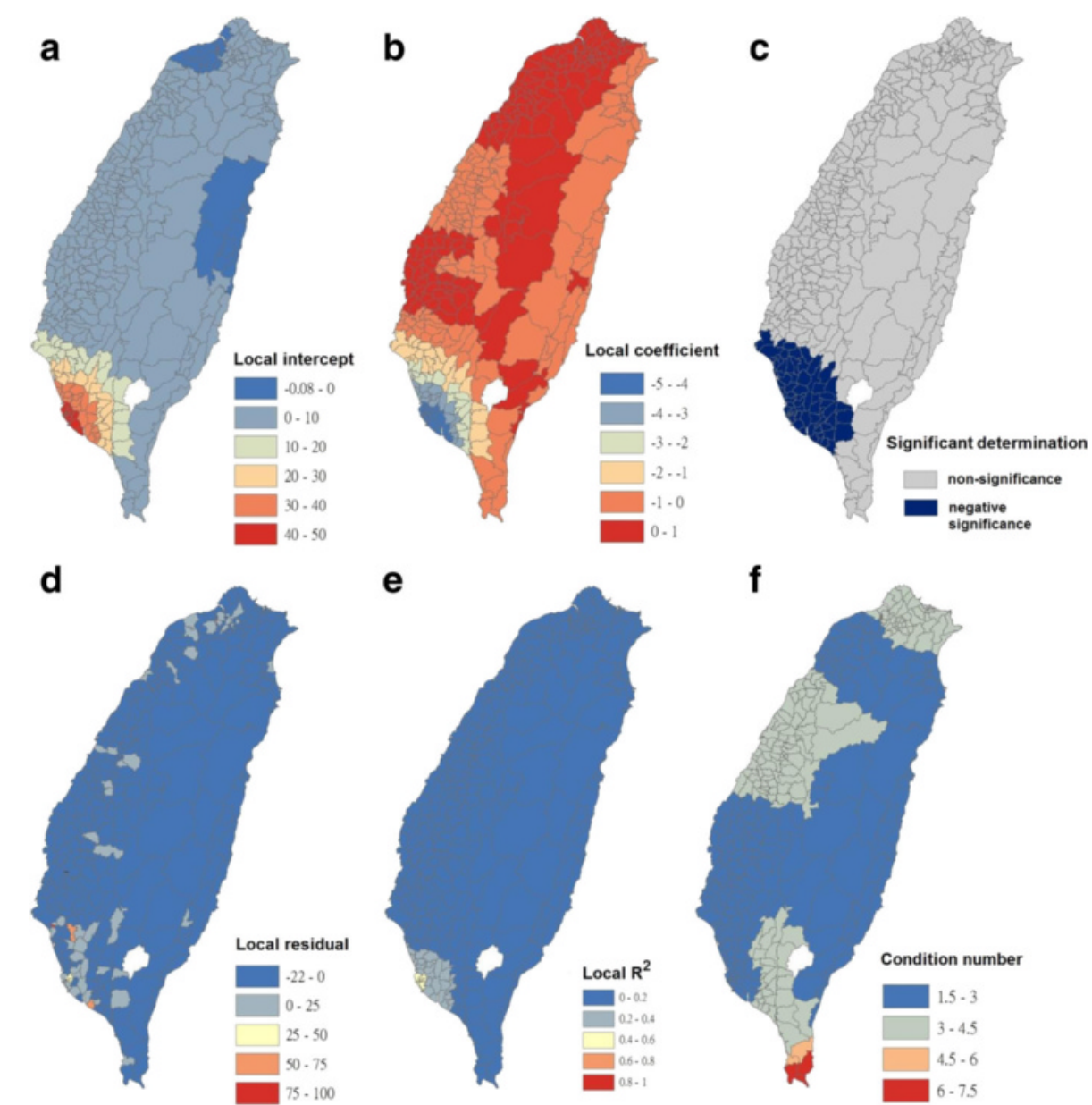

Fig. 6 Results of the GWR model for Breteau indices of Ae. albopictus (as the explanatory variable) and autochthonous incidence of dengue fever (as the response variable) in 348 townships in Taiwan during 2009-2011. a Local intercepts during 2009-2011. b Local regression coefficients during 2009-2011. c Significant determination of coefficients according to the Benjamini-Hochberg false discovery rate during 2009-2011. d Local residuals during 2009-2011. e Local R² values during 2009-2011. f Local condition number values during 2009-2011

Tropic of Cancer (i.e., $23.5^{\circ} \mathrm{N}$; local $\mathrm{R}^{2}<0.33$ ). The results indicated that the human population density contributed to the incidence of Ae. aegypti in southern Taiwan. We used bivariate Moran's I analysis to examine spatial dependence between the BI of $A e$. aegypti and density of human population, and the results were positively significant (bivariate Moran's index $=0.21$; Table 2 and Fig. 3h).

We used Yates' chi-square test to examine the patterns of confirmed dengue cases (imported or autochthonous) among 9 dengue outbreak years (i.e., 2002, 2006, 2007, 2009, 2010, 2011, 2012, 2014, and 2015); dengue outbreak years are defined as those having $>1000$ confirmed dengue cases. The two-sided test had a significance level of 0.05 . The results indicated that most of the years had dissimilar incidence patterns. However, few tests did not reject the null hypothesis, and the years with similar incidence patterns were classified into three groups: "2006, 2007, and
2011"; "2010 and 2012"; and "2002 and 2015," as shown in Table 3.

\section{Discussion}

Extensive and rapid urbanization without appropriate planning may have directly resulted in large numbers of artificial containers that are suitable for breeding Aedes mosquitoes around households. The rapidly developing air transportation has accelerated virus translocation, enabling it to easily migrate from endemic to non-endemic areas [54]. Therefore, the probability of virus introduction through imported cases has considerably increased, even in countries where dengue has never been reported. Moreover, travelers infected with DENV serve as vehicles for its potential transmission [55]. Other environmental factors, such as global warming, have been associated with the occurrence of dengue epidemics $[56,57]$, causing this disease to be more complicated and thus more difficult to manage. Socioeconomic 

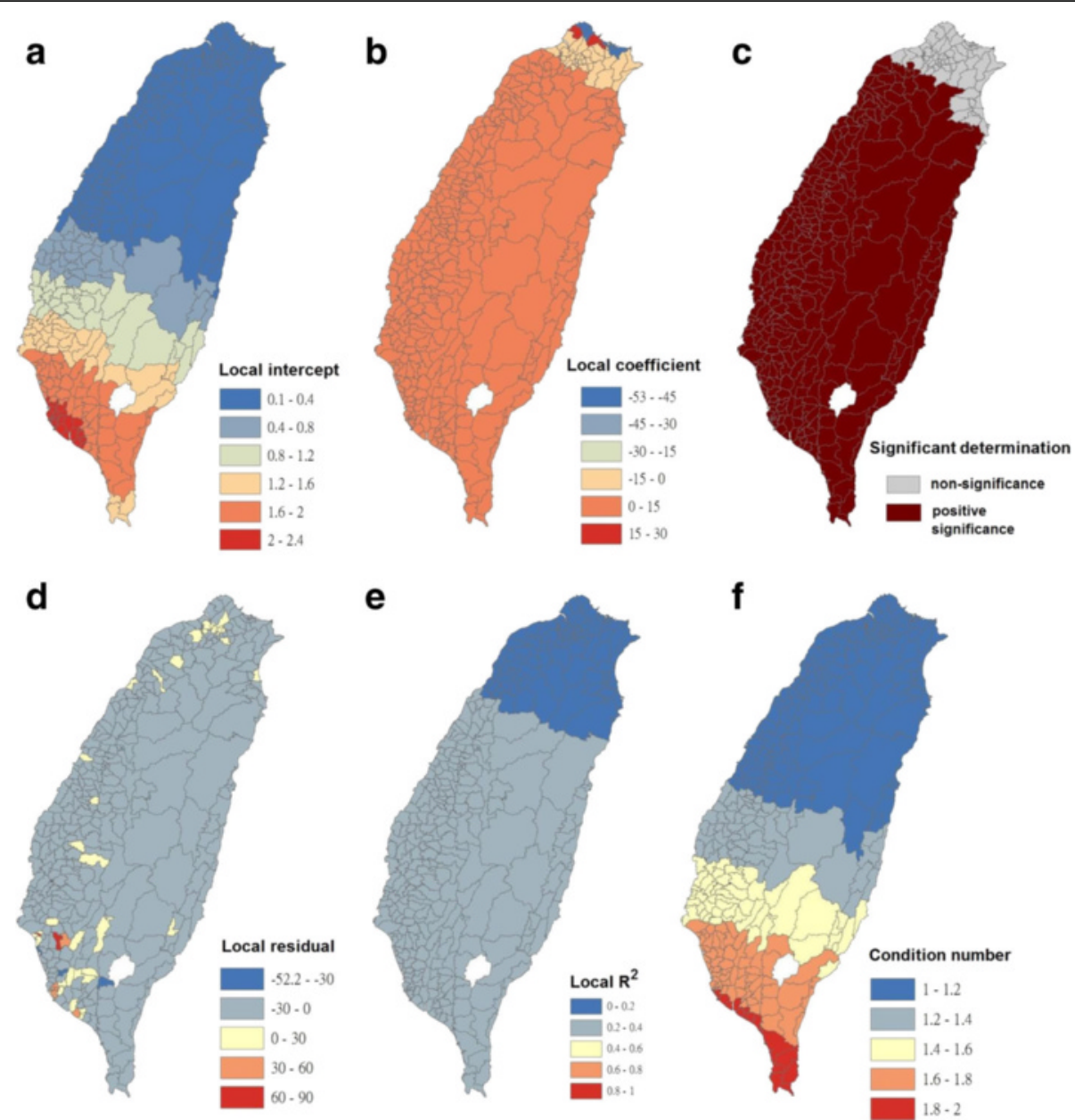

Fig. 7 Results of the GWR model for Breteau indices of Ae. aegypti (as the explanatory variable) and autochthonous incidence of dengue fever (as the response variable) in 348 townships in Taiwan during 2009-2011. a Local intercepts during 2009-2011. b Local regression coefficients during 2009-2011. c Significant determination of coefficients according to the Benjamini-Hochberg false discovery rate during 2009-2011. d Local residuals during 2009-2011. e Local $R^{2}$ values during 2009-2011. f Local condition number values during 2009-2011

factors affecting the distribution of Aedes mosquitoes, other than the use of containers to store water, include air conditioner use, housing quality, and urbanization rate $[58,59]$. Ae. aegypti and Ae. albopictus are found in both urban and rural areas. Ae. aegypti tends to breed in urbanized ecological niches, whereas Ae. albopictus is more frequently found in rural areas; however, mixed breeding has been reported [60]. In addition, Ae. aegypti prefers living indoors, whereas Ae. albopictus frequently breeds outdoors [61-63]. Dengue outbreaks have occurred nearly every year in Taiwan since 1987, first appearing in the southern part of Taiwan, which has a high human population density. Dengue outbreaks usually increase during rapid urbanization, and a high human population density has been associated with dengue transmission because of its potential impact on human-mosquito contact [64, 65]. Our study results revealed that the clusters of Ae. aegypti were associated with a high density of human population in the southern part of Taiwan, where the risk of dengue outbreaks is high (Figs. 2, 10, and 11). Ae. aegypti has more opportunities to increase human-mosquito contact and thus increase the probability of dengue outbreaks. However, the opposite results were observed for Ae. albopictus.

Studies on the spatial coexistence of Ae. aegypti and Ae. albopictus have reported that the two species are sympatric [66, 67]. In North America [68] and Brazil [66], the two species have similar larval ecological niches and often share the same larval habitat. Likewise, in Mayotte, Ae. albopictus coexists with Ae. aegypti in $40 \%$ of larval habitats [69]. However, as suggested by Paupy et al. [70], the apparent coexistence of the two species could be a transient situation, followed by a reduction [71-73] or displacement $[74,75]$ of the resident species; interspecific larval competition for resources is the most 

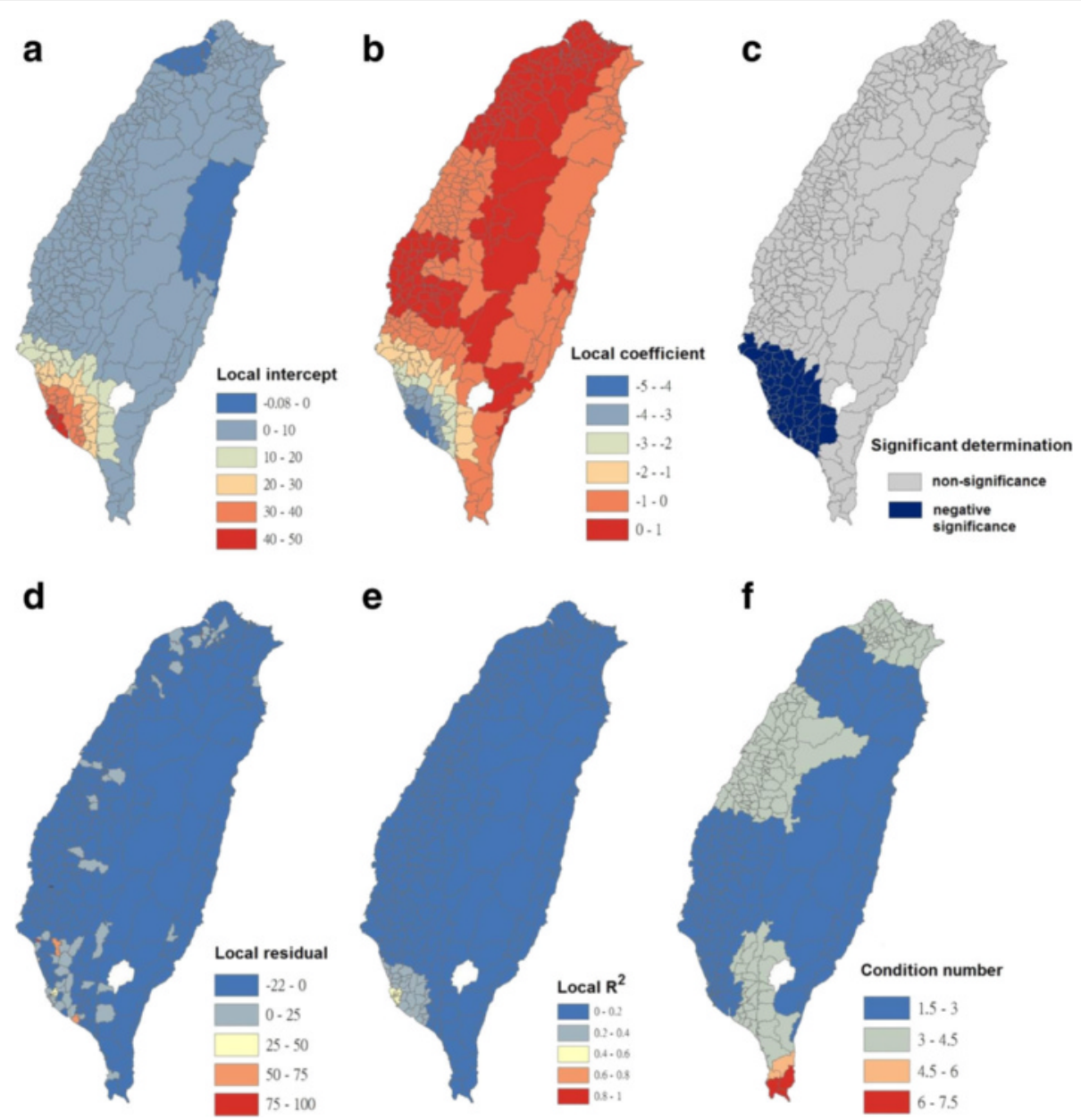

Fig. 8 Results of the GWR model for Breteau indices of Ae. albopictus (as the explanatory variable) and dengue fever incidence rates (as the response variable) in 348 townships in Taiwan during 2009-2011. a Local intercepts during 2009-2011. b Local regression coefficients during 2009-2011. c Significant determination of coefficients according to the Benjamini-Hochberg false discovery rate during 2009-2011. d Local residuals during 2009-2011. e Local R² values during 2009-2011. f Local condition number values during 2009-2011

likely reason for this process. Moreover, a few local studies have reported that the local spread of Ae. albopictus and decline in Ae. aegypti populations might be linked to interspecies competition $[68,76,77]$ or non-reciprocal cross-species inseminations [78].

Ae. aegypti almost exclusively feeds on humans in daylight hours and typically rests indoors [79]. By contrast, Ae. albopictus is usually exophagic and bites humans and animals opportunistically [70]; however, Ae. albopictus has been reported to exhibit strongly anthropophilic behavior similar to that of Ae. aegypti in specific contexts $[63,80]$. Both species inhabit containers but differ in their behavior and biology; thus, they occupy different niches [81]. Studies have reported that compared with Ae. aegypti, which is usually endophagic, Ae. albopictus tends to be exophagic and thus prefers to feed on blood indoors [62, 80]. Multiple blood feedings occur frequently in Ae. albopictus and Ae. Aegypti, which usually becomes engorged after two or three blood meals [80, 82-84]. However, multiple blood feedings in a single gonotrophic cycle are more frequent in Ae. aegypti [63, 82, 85]. Such behavioral differences may provide fewer opportunities for Ae. albopictus to contact humans infected by DENV. Thus, Ae. albopictus has a lower probability of transmitting the virus through the intake of a blood meal [86]. Mosquito behaviors are crucial in determining the occurrence and scale of many arthropod-borne diseases and diseases caused by nematodes, protozoans, and viruses [87].

Both Ae. albopictus and Ae. aegypti are susceptible to DENV. However, rates of salivary gland infection and transmission are higher in Ae. aegypti-fed blood meals than in Ae. albopictus-fed blood meals containing DENV, either in mosquitoes from Taiwan or Southeast Asian countries [86, 88]. A study on field-caught mosquitoes conducted in Singapore reported that $6.9 \%$ of 

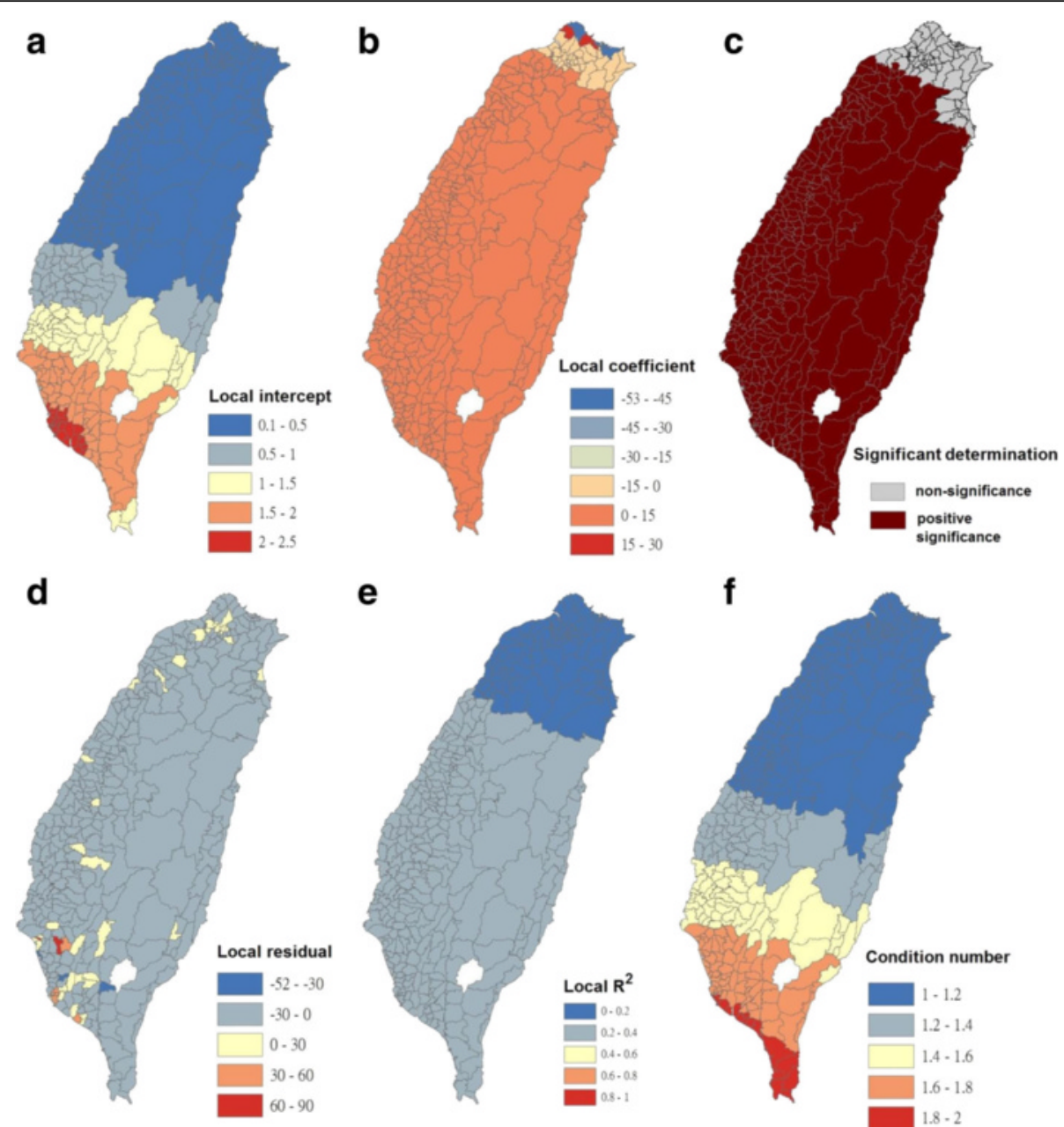

Fig. 9 Results of the GWR model for Breteau indices of Ae. aegypti (as the explanatory variable) and dengue fever incidence rates (as the response variable) in 348 townships in Taiwan during 2009-2011. a Local intercepts during 2009-2011. b Local regression coefficients during 2009-2011. c Significant determination of coefficients according to the Benjamini-Hochberg false discovery rate during 2009-2011. d Local residuals during 2009-2011. e Local R² values during 2009-2011. f Local condition number values during 2009-2011

Ae. aegypti, but only $2.9 \%$ of Ae. albopictus was positive for DENVs [89]. Another study conducted in Singapore reported that infected Ae. aegypti were detected as early as six weeks before the start of a dengue outbreak, whereas infected Ae. albopictus did not appear until the number of cases was increasing [21]. Furthermore, a study conducted in southern Taiwan demonstrated that DENV was detected in low levels only from $0.2 \%$ positive signs for field-caught Ae. aegypti, but not for Ae. albopictus [90]. Large outbreaks such as those in 2006 and 2007 occurred one year after the detection of virus-infected Ae. aegypti [90]. These results prompt the possibility that Ae. aegypti can efficiently establish a preliminary dengue case cluster. Because of frequent subclinical or cryptic infections, dengue outbreaks may be delayed or not reported, resulting in silent transmission [91]. In addition, competition for resources by other intracellular organisms is crucial for mosquitoes to support viral replication [92]. In Taiwan, the endosymbiont Wolbachia has been identified in nearly all Ae. albopictus populations; however, it is completely absent from Ae. aegypti populations breeding in the field [93]. The artificial introduction of an exogenous strain of Wolbachia, leading to competition for cellular resources, interfered with replication of DENV in Ae. aegypti. Wolbachia-mediated pathogen interference may synergistically work with the life-shortening strategy [92]. This suggests that this maternally inherited endosymbiont acts as a factor determining viral infection and its dissemination to other tissues; that is, from the midgut to the salivary glands of the mosquito [94, 95]. In addition, the study results in Malaysia revealed transovarial transmission of dengue virus in the larvae of Ae. aegypti and Ae. albopictus in the field, with $6.3 \%$ positive results [96]. However, according to a study of virological 

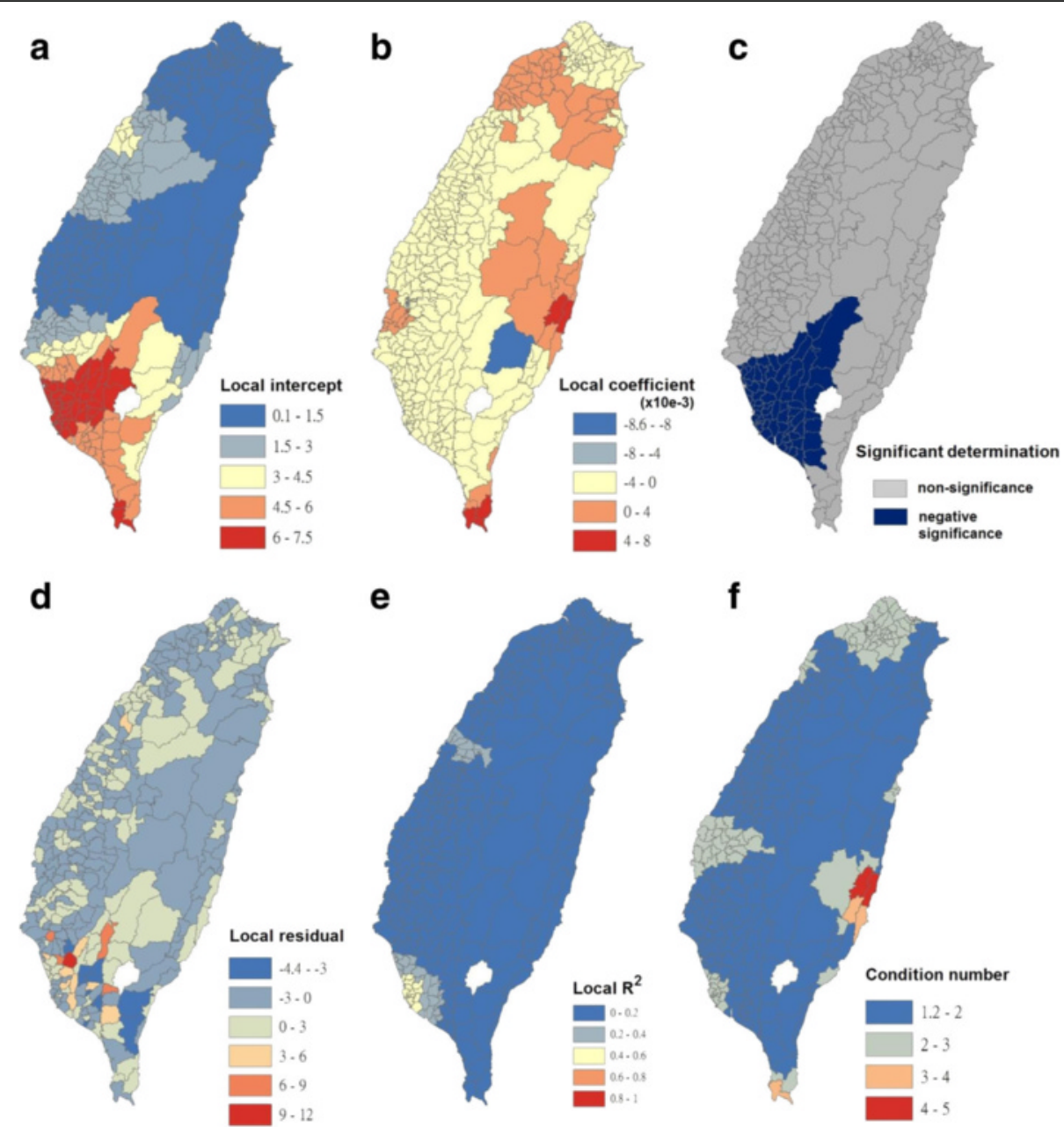

Fig. 10 Results of the GWR model for human population densities (as the explanatory variable) and Breteau indices of Ae. albopictus (as the response variable) in 348 townships in Taiwan during 2009-2011. a Local intercepts during 2009-2011. b Local regression coefficients during 2009-2011. c Significant determination of coefficients according to the Benjamini-Hochberg false discovery rate during 2009-2011. d Local residuals during 2009-2011. e Local $\mathrm{R}^{2}$ values during 2009-2011. f Local condition number values during 2009-2011

surveillance in Taiwan, transovarial transmission in local Aedes mosquitos may not occur, or occur at lower rates [90].

Since the epidemic in 1987, few small outbreaks have been reported in north of the Tropic of Cancer, such as those in Chungho (i.e., Jhonghe City) in Taipei County in 1995 (162 cases), Tunghai University in Taichung City in 1995 (8 cases), and Taipei City in 1996 (14 cases); these outbreaks occurred in areas without Ae. aegypti [97, 98]. Because Ae. albopictus is also susceptible to DENV [86, 99], it may also cause sporadic cases, and occasionally, small outbreaks. In turn, only a few autochthonous cases can occur and thus the scales of outbreaks are limited in northern Taiwan, although more imported cases are reported every year in that area. Despite similarities between Ae. aegypti and Ae. albopictus, they differ in crucial characteristics, including behaviors and vector competence to the dengue virus. Such differences may cause discrepancies in their roles in dengue outbreaks. Considering the occurrence of DF in past decades in Taiwan, only limited or no outbreaks can be established in which Ae. aegypti is absent (northern and central parts of Taiwan where Ae. albopictus is prevalent). Yang et al. (2014) [100] hypothesized that the existence of Ae. aegypti is a prerequisite for the initiation and establishment of a dengue outbreak, which may be expanded or maintained through participation of $A e$. albopictus. The outbreak may be terminated when herd immunity in the resident population reaches a level sufficiently high to block the spread of infections. However, this hypothesis is inconsistent with our results presented in Figs. 6 and 7. The clusters in dengue-endemic areas of southern Taiwan were located where the incidence of autochthonous cases was negatively correlated with $A e$. albopictus and positively correlated with Ae. aegypti. Our findings revealed that Ae. aegypti in endemic areas 


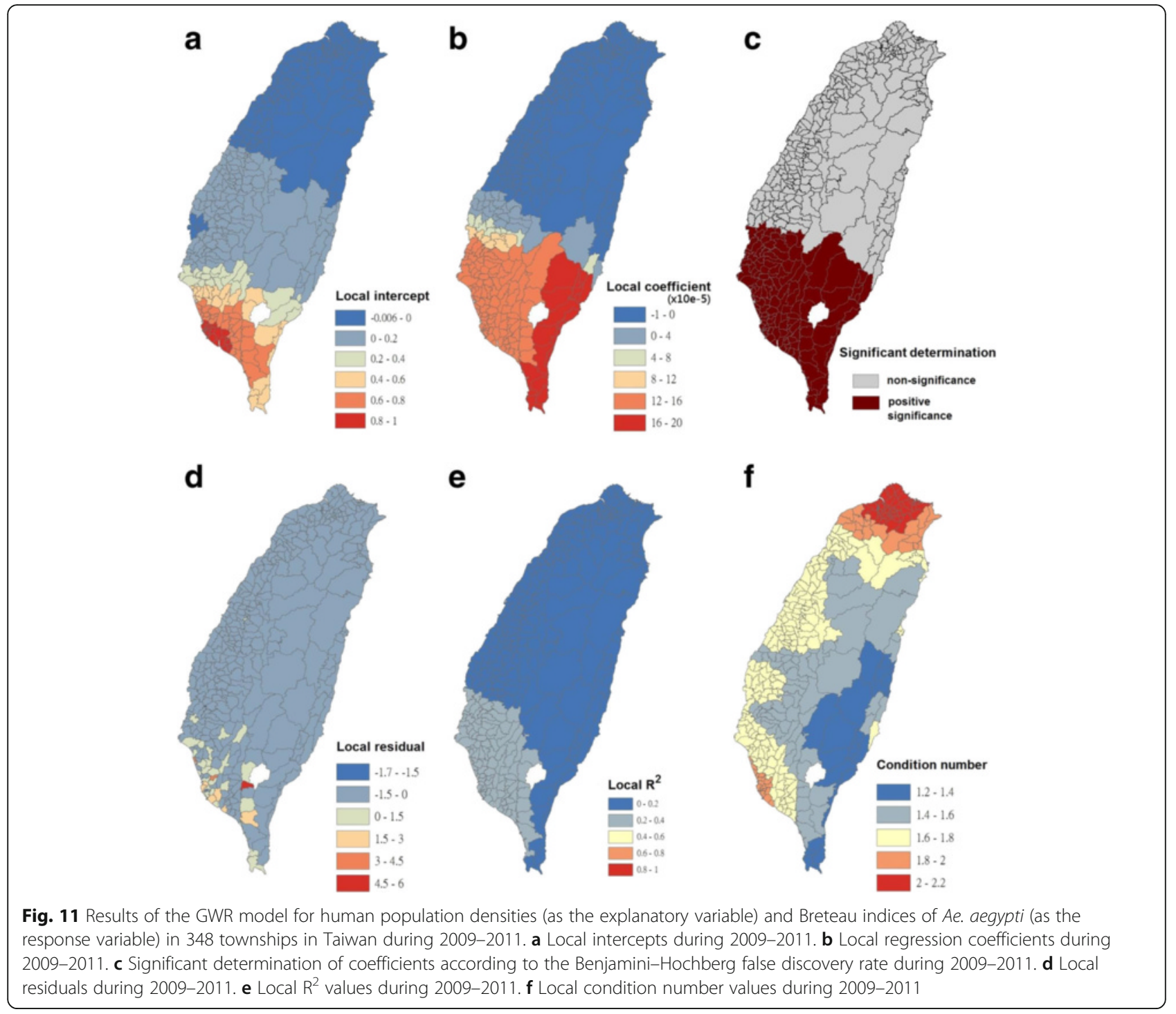

Table 3 Yates' chi-square test and matric comparisons of nine dengue outbreak years ${ }^{a}$

\begin{tabular}{|c|c|c|c|c|c|c|c|c|c|c|c|}
\hline Year & $\begin{array}{l}2002 \\
(\mathrm{Im} / \mathrm{Au})\end{array}$ & $\begin{array}{l}2006 \\
(\mathrm{Im} / \mathrm{Au})\end{array}$ & $\begin{array}{l}2007 \\
(\mathrm{Im} / \mathrm{Au})\end{array}$ & $\begin{array}{l}2009 \\
(\mathrm{Im} / \mathrm{Au})\end{array}$ & $\begin{array}{l}2010 \\
(\mathrm{Im} / \mathrm{Au})\end{array}$ & $\begin{array}{l}2011 \\
(\mathrm{Im} / \mathrm{Au})\end{array}$ & $\begin{array}{l}2012 \\
(\mathrm{Im} / \mathrm{Au})\end{array}$ & $\begin{array}{l}2014 \\
(\mathrm{Im} / \mathrm{Au})\end{array}$ & $\begin{array}{l}\text { Year } \\
2002\end{array}$ & $\begin{array}{l}\text { Imported case (Im) } \\
52\end{array}$ & $\begin{array}{l}\text { autochthonous case (Au) } \\
5336\end{array}$ \\
\hline $2006(\mathrm{Im} / \mathrm{Au})$ & *307.13 & & & & & & & & 2006 & 109 & 965 \\
\hline 2007 (Im/Au) & *273.09 & 3.1 & & & & & & & 2007 & 179 & 2000 \\
\hline 2009 (Im/Au) & *778.08 & *35.43 & *83.76 & & & & & & 2009 & 204 & 848 \\
\hline $2010(\mathrm{Im} / \mathrm{Au})$ & *681.82 & *19.34 & *58.58 & *5.12 & & & & & 2010 & 304 & 1592 \\
\hline 2011 (Im/Au) & *305.53 & 0.55 & 1.11 & *58.12 & *36.62 & & & & 2011 & 157 & 1545 \\
\hline $2012(\mathrm{Im} / \mathrm{Au})$ & *539.76 & *8.17 & *30.67 & $* 12.71$ & 2.5 & *17.37 & & & 2012 & 207 & 1271 \\
\hline $2014(\mathrm{Im} / \mathrm{Au})$ & *8.84 & *363.42 & *371.93 & *1215.21 & *1186.02 & *405.63 & *335.02 & & 2014 & 240 & 15492 \\
\hline $2015(\mathrm{Im} / \mathrm{Au})$ & 0.84 & ${ }^{*} 861.18$ & *960.62 & *2808.97 & *2899.05 & *1009.39 & *1242.15 & *54.38 & 2015 & 365 & 43419 \\
\hline
\end{tabular}

Im imported case, $A u$ autochthonous case

Significance $(*)$ is a chi-square value $>3.84(p$ value $=0.05)$

aindicates the number of confirmed dengue $>1000$ cases

Data extracted from Taiwan CDC [37] 
played a critical role in establishing most scales of dengue outbreaks during 2009-2011. However, Ae. albopictus may have a supporting role or cause sporadic cases and occasionally small outbreaks in the absence of Ae. aegypti.

\section{Conclusions}

Ae. aegypti is a key vector for dengue epidemics in Taiwan. According to the BIs of Ae. aegypti, but not of Ae. albopictus, high-density clusters of human populations are highly associated with the emergence of dengue epidemics in southern Taiwan. Ae. albopictus may have a supporting role during small-scale dengue outbreaks or cause sporadic cases and occasionally small outbreaks in the absence of Ae. aegypti. This observation may provide broader considerations for health authorities responsible for dengue prevention, hopefully leading to a more efficient allocation of resources, particularly in the control of mosquito vectors.

\section{Acknowledgments}

The authors thank the CDC for providing access to the database on notifiable infectious diseases and related statistics on infectious diseases. The authors also thank the Ministry of the Interior for providing access to the demographic data.

\section{Funding}

No funding was obtained for this study.

\section{Availability of data and materials}

All the data supporting the findings is contained within the manuscript.

\section{Authors' contributions}

PJ was responsible for the study design, epidemiological enquiry, data collection, statistical calculations, and drafting of the manuscript. HJ assisted with the collection of Aedes mosquito data. Both authors read and approved the final manuscript.

\section{Competing interests}

The authors declare that they have no competing interests.

\section{Consent for publication}

Not applicable.

Ethics approval and consent to participate

Not applicable.

\section{Author details}

${ }^{1}$ Center for General Education, Aletheia University, New Taipei City 25103, Taiwan, ROC. ${ }^{2}$ Center for Diagnostics and Vaccine Development, Centers for Disease Control, Taipei, Taiwan, ROC.

Received: 30 May 2016 Accepted: 28 October 2016

Published online: 09 November 2016

\section{References}

1. Bhatt S, Gething PW, Brady OJ, Messina JP, Farlow AW, Moyes CL, et al. The global distribution and burden of dengue. Nature. 2013;496(7446):504-7.

2. Armstrong C. Dengue fever. Pub Health Rep. 1923;38:1750-84.

3. Rigau-Pérez JG, Clark GG, Gubler DJ, Reiter P, Sanders EJ, Vorndam AV. Dengue and dengue haemorrhagic fever. Lancet. 1998;352:971-7.

4. Goh KT, Ng SK, Chan YC, Lim SJ, Chua EC. Epidemiological aspects of an outbreak of dengue fever/dengue haemorrhagic fever in Singapore. Southeast Asian J Trop Med Pub Health. 1987;18:291-4.
5. Schaffner F, Mathis A. Dengue and dengue vectors in the WHO European region: past, present, and scenarios for the future. Lancet Infect Dis. 2014; 14(12):1271-80.

6. Brady OJ, Gething PW, Bhatt S, Messina JP, Brownstein JS, Hoen AG, et al. Refining the global spatial limits of dengue virus transmission by evidencebased consensus. PLoS Negl Trop Dis. 2012;6(8):e1760.

7. Simmons CP, Farrar JJ. Nguyen vV, Wills B. Current concept: dengue. N Engl J Med. 2012;366:1423-32.

8. Noisakran S, Perng GC. Alternate hypothesis on the pathogenesis of dengue hemorrhagic fever (DHF)/dengue shock syndrome (DSS) in dengue virus infection. Exp Biol Med (Maywood). 2008;233:401-8.

9. Sam SS, Omar SF, Teoh BT, Abd-Jamil J, AbuBakar S. Review of dengue hemorrhagic fever fatal cases seen among adults: a retrospective study. PLoS Negl Trop Dis. 2013;7(5):e2194.

10. Rudnick A, Marchette NJ, Garcia R. Possible jungle dengue - recent studies and hypotheses. Jpn J Med Sci Biol. 1967;20:69-74.

11. Teoh BT, Sam SS, Abd-Jamil J, AbuBakar S. Isolation of ancestral sylvatic dengue virus type 1, Malaysia. Emerg Infect Dis. 2010;16:1783-85.

12. Calisher CH, Karabatsos N, Dalrymple JM, Shope RE, Porterfield JS, Westaway EG, et al. Antigenic relationships between flaviviruses as determined by cross-neutralization tests with polyclonal antisera. J Gen Virol. 1989;70:37-43.

13 Salazar MI, Richardson JH, Sánchez-Vargas I, Olson KE, Beaty BJ. Dengue virus type 2: replication and tropisms in orally infected Aedes aegypti mosquitoes. BMC Microbiol. 2007;7:9.

14 Reinert JF, Harbach RE, Kitching IJ. Phylogeny and classification of tribe Aedini (Diptera: Culicidae). Zool J Linn Soc. 2009. doi:10.1111/j.1096-3642. 2009.00570.x.

15 Simmons CP, Farrar JJ, Chau NW, Wills B. Dengue. N Engl J Med. 2012. doi: 10.1056/NEJMra1110265

16 Jentes ES, Poumerol G, Gershman MD, Hill DR, Lemarchand J, Lewis RF, et al. The revised global yellow fever risk map and recommendations for vaccination, 2010: consensus of the informal WHO Working Group on Geographic Risk for Yellow Fever. Lancet Infect Dis. 2011;11(8):622-32.

17 Leparc-Goffart I, Nougairede A, Cassadou S, Prat C, de Lamballerie X. Chikungunya in the Americas. Lancet. 2014;383(9916):514.

18 Rodriguez-Morales AJ, Bandeira AC, Franco-Paredes C. The expanding spectrum of modes of transmission of Zika virus: a global concern. Ann Clin Microbiol Antimicrob. 2016:15:13.

19 Brown JE, Evans BR, Zheng W, Obas V, Barrera-Martinez L, Egizi A, et al. Human impacts have shaped historical and recent evolution in Aedes aegypti, the dengue and yellow fever mosquito. Evolution. 2014;68(2):514-25.

20 Brown JE, McBride CS, Johnson P, Ritchie S, Paupy C, Bossin H, et al. Worldwide patterns of genetic differentiation imply multiple 'domestications' of Aedes aegypti, a major vector of human diseases. Proc Biol Sci. 2011;278(1717):2446-54.

21 Chow VTK, Chan YC, Yong R, Lee KM, Lim LK, Chung YK, et al. Monitoring of dengue viruses in field-caught Aedes aegypti and Aedes albopictus mosquitoes by a type-specific polymerase chain reaction and cycle sequencing. Am J Trop Med Hyg. 1998;58(5):578-86.

22 Delatte AH, Gimonneau G, Triboire A, Fontenille D, Delatte H. Influence of temperature on immature development, survival, longevity, fecundity, and gonotrophic cycles of Aedes albopictus, vector of chikungunya and dengue in the Indian Ocean. J Med Entomol. 2009;46(1):33-41.

23 Medlock JM, Hansford KM, Schaffner F, Versteirt V, Hendrickx G, Zeller H, et al. A review of the invasive mosquitoes in Europe: ecology, public health risks, and control options. Vector Borne Zoonotic Dis. 2012;12(6):435-47.

24 Carvalho RG, Lourenc, o-de-Oliveira R, Braga IA. Updating the geographical distribution and frequency of Aedes albopictus in Brazil with remarks regarding its range in the Americas. Mem Inst Oswaldo Cruz. 2014;109(6):787-96.

25 Ngoagouni C, Kamgang B, Nakouné E, Paupy C, Kazanji M. Invasion of Aedes albopictus (Diptera: Culicidae) into central Africa: what consequences for emerging diseases? Parasit Vectors. 2015;8:191.

26 Lin TH. Surveillance and control of Aedes aegypti in epidemic areas of Taiwan. Kaohsiung J Med Sci. 1994;10(Suppl):S88-93.

27 Lin C, Wang CY, Teng HJ. The study of dengue vector distribution in Taiwan from 2009 to 2011. Taiwan Epidemiol Bull. 2014;30(15):304-10 (in Chinese).

28 Chang LH, Hsu EL, Teng HJ, Ho CM. Aedes albopictus (Diptera: Culicidae) larvae exposed to low temperatures in Taiwan. J Med Entomol. 2007;44(2): 205-10.

29 Giménez JO, Fischer S, Zalazar L, Stein M. Cold season mortality under natural conditions and subsequent hatching response of Aedes (Stegomyia) 
aegypti (Diptera: Culicidae) eggs in a subtropical city of Argentina. J Med Entomol. 2015;52(5):879-85.

30 Lin CC, Huang YH, Shu PY, Wu HS, Lin YS, Yeh TM, et al. Characteristic of dengue disease in Taiwan: 2002-2007. Am J Trop Med Hyg. 2010;82(4):731-9.

31 Shu PY, Su CL, Liao TL, Yang CF, Chang SF, Lin CC, et al. Molecular characterization of dengue viruses imported into Taiwan during 2003-2007: geographic distribution and genotype shift. Am J Trop Med Hyg. 2009;80(6): 1039-46.

32 Huang JH, Su CL, Yang CF, Liao TL, Hsu TC, Chang SF, et al. Molecular characterization and phylogenetic analysis of dengue viruses imported into Taiwan during 2008-2010. Am J Trop Med Hyg. 2012;87(2):349-58.

33 van Panhuis WG, Choisy M, Xiong X, Chok NS, Akarasewi P, lamsirithaworn $S$, et al. Region-wide synchrony and traveling waves of dengue across eight countries in Southeast Asia. Proc Natl Acad Sci U S A. 2015;112(42):13069-74.

34 Bureau of Communicable Disease Control. Preliminary investigation report of an outbreak of dengue fever in Kaohsiung and Pingtung, southern Taiwan. Epidemiol Bull. 1987;1987(Dec):93-5.

35 Wu YC. Epidemic dengue 2 on Liouchyou Shaing, Pingtung County in 1981. Chinese J Microbiol Immunol. 1986;19:203-11.

36 King CC, Wu YC, Chao DY, Lin TH, Chow L, Wang HT, et al. Major epidemics of dengue in Taiwan in 1981-2000: related to intensive virus activities in Asia. Dengue Bulletin. 2000;24:1-10.

37 Taiwan National Infectious Disease Statistics System. http://nidss.cdc.gov.tw/ en/?treeid=00ED75D6C887BB27\&nowtreeid=D39475C2DB7CD87B. 2016. Accessed 22 Jan 2016.

38 Dengue Control, World Health Organization (WHO). http://www.who.int/ denguecontrol/monitoring/vector_surveillance/en/. 2016. Accessed 4 Sep 2016.

39 Centers for Disease Control, R.O.C. (Taiwan). Guidelines for dengue/ chikungunya control. 7th ed. Taipei: Centers for Disease Control, R.O.C. (Taiwan); 2014

40 Monthly Bulletin of Interior Statistics. http://sowf.moi.gov.tw/stat/month/list. htm. 2015. Accessed 13 Oct 2015

41 Boots BN, Getis A. Point pattern analysis. Newbury Park: Sage Publications; 1998.

42 Cliff AC, Ord JK. Spatial autocorrelation. London: Pion Limited; 1973.

43 Legendre P, Legendre L. Numerical ecology. 2nd ed. Amsterdam: Elsevier; 1998.

44 Grubesic TH. Zip codes and spatial analysis: problems and prospects. Socio Econ Plan Sci. 2008:42:129-49.

45 Cliff AD, Ord JK. Spatial processes: models and applications. London: Pion Limited; 1981.

46 Anselin L, Syabri I, Smirnov O. Visualizing multivariate spatial correlation with dynamically linked windows. Santa Barbara: University of California; 2002. CD-ROM. http://citeseerx.ist.psu.edu/viewdoc/summary?doi=10.1.1.118. 7163. Accessed 5 Sep 2016

47 Wartenberg D. Multivariate spatial correlation: a method for exploratory geographical analysis. Geogr Anal. 1985;17:263-83.

48 GeoDa 1.4.6. https://spatial.uchicago.edu/software (2016). Accessed 5 Sep 2016.

49 Fotheringham AS, Brunsdon C, Charlton M. Geographically weighted regression: the analysis of spatially varying relationships. Wiley: Chichester; 2002.

50 Fotheringham AS, Brunsdon C, Charlton M. Geographically weighted regression: a natural evolution of the expansion method for spatial data analysis. Environ Planning A. 1998;30:1905-27.

51 Charlton M, Fotheringham AS. Geographically weighted regression (White Paper). National Centre for Geocomputation National University of Ireland, Maynooth. http://www.geos.ed.ac.uk/ gisteac/fspat/gwr/gwr_arcgis/GWR WhitePaper.pdf. 2009. Accessed 17 Sep 2016.

52 Thissen D, Steinberg L, Kuang D. Quick and easy implementation of the Benjamini-Hochberg procedure for controlling the false positive rate in multiple comparisons. J Educ Behav Stat. 2002;27:77-83.

53 Rosner B. Hypothesis testing: on sample inference. In: Fundamentals of biostatistics. 7th ed. Boston: Brooks/Cole, Cengage Learning; 2011. p. 204-57.

54 Gubler DJ. Epidemic dengue/dengue hemorrhagic fever as a public health, social and economic problem in the 21st century. Trends Microbiol. 2002;10:100-3

55 Jelinek T. Dengue fever in international travelers. Clin Infect Dis. 2000; 31(1):144-7.

56 Herrera-Martinez AD, Rodríguez-Morales AJ. Potential influence of climate variability on dengue incidence registered in a western pediatric hospital of Venezuela. Trop Biomed. 2010;27(2):280-6.
57 Liu-Helmersson J, Stenlund H, Wilder-Smith A, Rocklöv J. Vectorial capacity of Aedes aegypti: effects of temperature and implications for global dengue epidemicpotential. PLoS One. 2014;9(3):e89783.

58 Ramos MM, Mohammed H, Zielinski-Gutierrez E, Hayden MH, Lopez JLR, Fournier $M$, et al. Epidemic dengue and dengue hemorrhagic fever at the Texas-Mexico border: results of a household-based seroepidemiologic survey, December 2005. Am J Trop Med Hyg. 2008;78(3):364-9.

59 Aström C, Rocklöv J, Hales S, Béguin A, Louis V, Sauerborn R. Potential distribution of dengue fever under scenarios of climate change and economic development. Ecohealth. 2012;9(4):448-54.

60 Calderón-Arguedas O, Troyo A, Solano ME, Avendano A, Beier JC. Urban mosquito species (Diptera: Culicidae) of dengue endemic communities in the Greater Puntarenas area. Rev Biol Trop. 2009;57(4):1223-34.

61 Hwang JS, Hsu EL, Chen YR. Investigations on the density and breeding habitats of Aedes mosquitoes in dengue epidemic areas in Taiwan. Chin J Pub Health (Taiwan). 1995;14:228-36.

62 Thavara U, Tawatsin A, Chansang C. Larval occurrence, oviposition behavior and biting activity of potential mosquito vectors of dengue on Samui Island. Thailand J Vector Ecol. 2001:26(2):172-80.

63 Ponlawat A, Harrington LC. Blood feeding patterns of Aedes aegypti and Aedes albopictus in Thailand. J Med Entomol. 2005:42(5):844-9.

64 Padmanabha H, Durham D, Correa F, Diuk-Wasser M, Galvani A. The interactive roles of Aedes aegypti super-production and human density in dengue transmission. PLoS Negl Trop Dis. 2012;6(8):e1799.

65 Rodrigues Mde M, Marques GR, Serpa LL, Arduino Mde B, Voltolini JC, Barbosa GL, et al. Density of Aedes aegypti and Aedes albopictus and its association with number of residents and meteorological variables in the home environment of dengue endemic area, São Paulo, Brazil. Parasit Vectors. 2015;8:115.

66 Braks MA, Honorio NA, Lourencqo-De-Oliveira R, Juliano SA, Lounibos LP. Convergent habitat segregation of Aedes aegypti and Aedes albopictus (Diptera: Culicidae) in southeastern Brazil and Florida. J Med Entomol. 2003; 40(6):785-94.

67 Chen CD, Nazni WA, Lee HL, Seleena B, Mohd Masri S, Chiang YF, et al. Mixed breeding of Aedes aegypti (L.) and Aedes albopictus Skuse in four dengue endemic areas in Kuala Lumpur and Selangor, Malaysia. Trop Biomed. 2006;23(2):224-7.

68 Juliano SA, Lounibos LP, O'Meara GF. A field test for competitive effects of Aedes albopictus on A. aegypti in South Florida: differences between sites of coexistence and exclusion? Oecologia. 2004;139(4):583-93.

69 Bagny L, Freulon M, Delatte H. First record of Aedes albopictus, vector of arboviruses in the Eparse Islands of the Mozambique Channel and updating of the inventory of Culicidae. Bull Soc Pathol Exot. 2009;102(3):193-8.

70 Paupy C, Delatte H, Bagny L, Corbel V, Fontenille D. Aedes albopictus, an arbovirus vector: from the darkness to the light. Microbes Infect. 2009; 11(14-15):1177-85.

71 Bagny L, Delatte H, Quilici S, Fontenille D. Progressive decrease in Aedes aegypti distribution in Reunion Island since the 1900s. J Med Entomol. 2009; 46(6):1541-5.

72 Bagny L, Delatte H, Elissa N, Quilici S, Fontenille D. Aedes (Diptera: Culicidae) vectors of arboviruses in Mayotte (Indian Ocean): distribution area and larval habitats. J Med Entomol. 2009;46(2):198-207.

73 Raharimalala FN, Ravaomanarivo LH, Ravelonandro P, Rafarasoa LS, Zouache K, Tran-Van V, et al. Biogeography of the two major arbovirus mosquito vectors, Aedes aegypti and Aedes albopictus (Diptera, Culicidae), in Madagascar. Parasit Vectors. 2012;5:56

74 Lounibos LP. Invasions by insect vectors of human disease. Annu Rev Entomol. 2002:47:233-66.

75 Juliano SA, Lounibos LP. Ecology of invasive mosquitoes: effects on resident species and on human health. Ecol Lett. 2005;8(5):558-74.

76 O'Meara GF, Evans LF, Gettman AD, Cuda JP. Spread of Aedes albopictus and decline of Ae. aegypti (Diptera: Culicidae) in Florida. J Med Entomol. 1995; 32(4):554-62.

77 Daugherty MP, Alto BW, Juliano SA. Invertebrate carcasses as a resource for competing Aedes albopictus and Aedes aegypti (Diptera: Culicidae). J Med Entomol. 2000;37(3):364-72.

78 Bargielowski IE, Lounibos LP, Carrasquilla MC. Evolution of resistance to satyrization through reproductive character displacement in populations of invasive dengue vectors. Proc Natl Acad Sci U S A. 2013;110(8):2888-92.

79 Scott TW, Takken W. Feeding strategies of anthropophilic mosquitoes result in increased risk of pathogen transmission. Trends Parasitol. 2012;28(3):114-21. 
80 Delatte H, Desvars A, Bou'etard A, Bord S, Gimonneau G, Vourc'h G, et al. Blood-feeding behavior of Aedes albopictus, a vector of chikungunya on La Reunion. Vector Borne Zoonotic Dis. 2010;10(3):249-58.

81 Eisen L, Moore CG. Aedes (Stegomyia) aegypti in the continental United States: a vector at the cool margin of its geographic range. J Med Entomol. 2013:50(3):467-78.

82 Scott TW, Clark GG, Lorenz LH, Amerasinghe PH, Reiter P, Edman JD. Detection of multiple blood feeding in Aedes aegypti (Diptera: Culicidae) during a single gonotrophic cycle using a histologic technique. J Med Entomol. 1993;30(1):94-9.

83 Xue RD, Edman JD, Scott TW. Age and body size effects on blood meal size and multiple blood feeding by Aedes aegypti (Diptera: Culicidae). J Med Entomol. 1995;32(4):471-4.

84 Xue RD, Barnard DR, Ali A. Influence of multiple blood meals on gonotrophic dissociation and fecundity in Aedes Albopictus. J Am Mosa Control Assoc. 2009;25(4):504-7.

85 Canyon DV, Hii JL, Muller R. The frequency of host biting and its effect on oviposition and survival in Aedes aegypti (Diptera: Culicida). J Med Entomol. 1999:36(3):301-8.

86 Chen WJ, Wei HL, Hsu EL, Chen ER. Vector competence of Aedes albopictus and Ae. aegypti (Diptera: Culicidae) to dengue 1 virus in Taiwan: development of the virus in the orally and parenterally infected mosquitoes. J Med Entomol. 1993;30(3):524-30.

87 Day JF. Host-seeking strategies of mosquito disease vectors. J Am Mosq Control Assoc. 2005;21 (4 Suppl):17-22.

88 Vazeille M, Rosen L, Mousson L, Failloux AB. Low oral receptivity for dengue type 2 viruses of Aedes albopictus from Southeast Asia compared with that of Aedes aegypti. Am J Trop Med Hyg. 2003;68(2):203-8.

89 Chung YK, Pang FY. Dengue virus infection rate in field populations of female Aedes aegypti and Aedes albopictus in Singapore. Trop Med Int Health. 2002;7(4):322-30.

90 Chen CF, Shu PY, Teng HJ, Su CL, Wu JW, Wang JH, et al. Screening of dengue virus in field-caught Aedes aegypti and Aedes albopictus (Diptera: Culicidae) by one-step SYBR green-based reverse transcriptase-polymerase chain reaction assay during 2004-2007 in Southern Taiwan. Vector Borne Zoonotic Dis. 2010;10(10):1017-25.

91 Chen WJ, Chen SL, Chien LJ, Chen CC, King CC, Harn MR, et al. Silent transmission of the dengue virus in southern Taiwan. Am J Trop Med Hyg. 1996:55(1):12-6.

92 Moreira LA, Iturbe-Ormaetxe I, Jeffery JA, Lu G, Pyke AT, Hedges LM, et al. A Wolbachia symbiont in Aedes aegypti limits infection with dengue, Chikungunya, and Plasmodium. Cell. 2009;139(7):1268-78.

93 Tsai KH, Huang CG, Lien JC, Wu WJ, Chen WJ. Molecular (sub) grouping of the endosymbiont Wolbachia infection among mosquitoes of Taiwan. J Med Entomol. 2004;41(4):677-83.

94 Lu P, Bian G, Pan X, Xi Z. Wolbachia induces density-dependent inhibition to dengue virus in mosquito cells. PLoS Negl Trop Dis. 2012;6(7):e1754.

95 Mousson L, Zouache K, Arias-Goeta C, Raquin V, Mavingui P, Failloux AB. The native Wolbachia symbionts limit transmission of dengue virus in Aedes albopictus. PLoS Negl Trop Dis. 2012;6(12):e1989.

96 Rohani A, Aidil Azahary AR, Malinda M, Zurainee MN, Rozilawati H, Wan Najdah WM, et al. Eco-virological survey of Aedes mosquito larvae in selected dengue outbreak areas in Malaysia. J Vector Borne Dis. 2014;51(4): 327-32.

97 Department of Health $(\mathrm{DOH})$. Report cases of infectious diseases in Taiwan. Health Rep. 1996;13:1-406 (in Chinese).

98 Wu YC. Recent outbreaks in Taiwan area. Health Rep. 1996;6:2-6 (in Chinese).

99 Gratz NG. Critical review of the vector status of Aedes albopictus. Med Vet Entomol. 2004;18(3):215-27.

100 Yang CF, Hou JN, Chen TH, Chen WJ. Discriminable roles of Aedes aegypti and Aedes albopictus in establishment of dengue outbreaks in Taiwan. Acta Trop. 2014;130:17-23.

\section{Submit your next manuscript to BioMed Central and we will help you at every step:}

- We accept pre-submission inquiries

- Our selector tool helps you to find the most relevant journal

- We provide round the clock customer support

- Convenient online submission

- Thorough peer review

- Inclusion in PubMed and all major indexing services

- Maximum visibility for your research

Submit your manuscript at www.biomedcentral.com/submit
C) Biomed Central 